\title{
Outage Analysis for Coherent Decode-Forward Relaying Over Rayleigh Fading Channels
}

\author{
Ahmad Abu Al Haija, Student Member, IEEE, and Mai Vu, Senior Member, IEEE
}

\begin{abstract}
We analyze the outage performance of coherent partial decode-forward (pDF) relaying over Rayleigh fading channels in both half- and full-duplex transmissions. In coherent DF relaying, the relay either partially or fully decodes the source message, then coherently forwards the decoded message with the source to the destination. This coherent transmission creates a beamforming gain from the source and relay to the destination which improves the transmission rate. We analyze the impact of this beamforming gain on the outage performance, considering outage events at both the relay and the destination. We derive analytical expressions for the overall outage probability, assuming full CSI at receivers and only limited CSI at transmitters. We further show that at high SNR, coherent $\mathrm{pDF}$ relaying converges to full DF relaying and achieves the full diversity order of 2 . These analyses provide a fundamental understanding of the reliability of coherent pDF relaying and form the basis for analyzing performance in larger network settings. Numerical results show that partial decoding at the relay outperforms full decoding for low SNR and high target rates in the half-duplex mode. Comparison with existing results further shows that source-relay coherent transmission and joint decoding at the destination both improve the outage performance.
\end{abstract}

Index Terms-Decode-forward relaying, coherent relaying, partial relaying, joint decoding, outage analysis, diversity order.

\section{INTRODUCTION}

$\mathbf{C}$ OOPERATIVE communication can be deployed as an efficient method to improve network performance in modern wireless networks including ad hoc and cellular systems [1]. Relaying is a technique that has received increasing attention as a basis for cooperative communication. A majority of works on the relay channel have focused on the transmission scheme and the achievable rate. Practical services, however, often require a specific rate to be sustained, which necessitates the understanding of outage performance. Existing outage performance results are mostly derived for non-coherent and full decodeforward (DF) relaying, even though coherent and partial DF (pDF) relaying can significantly improve the achievable rate and outage performance. Comprehensive formulation and anal-

Manuscript received August 13, 2014; revised December 17, 2014 and January 29, 2015; accepted January 29, 2015. Date of publication February 10, 2015; date of current version April 14, 2015. This work has been supported in part by grants from the Natural Science and Engineering Research Council of Canada (NSERC) and the Fonds Québécois de la Recherche sur la Nature et les Technologies (FQRNT). The associate editor coordinating the review of this paper and approving it for publication was T. Tsiftsis.

A. Abu Al Haija is with the Department of Electrical and Computer Engineering, McGill University, Montreal, QC H3A 0E9, Canada (e-mail: ahmad.abualhaija@mail.mcgill.ca).

$\mathrm{M}$. Vu is with the Department of Electrical and Computer Engineering, Tufts University, Medford, MA 02155 USA (e-mail: maivu@ece.tufts.edu).

Color versions of one or more of the figures in this paper are available online at http://ieeexplore.iee.org.

Digital Object Identifier 10.1109/TCOMM.2015.2402154 ysis of coherent pDF outage performance are of interest for wireless applications.

\section{A. Background and Motivation}

Even though basic relaying techniques were developed since [2], [3] including decode, compress and amplify-forward techniques, interest in the application of relaying in wireless communications did not emerge until much more recently [4]-[8]. In this paper, we will only focus on decode-forward based schemes.

Outage probability has been analyzed for several decodeforward based schemes in [6]-[27]. Most of these works, however, did not consider coherent transmission from the source and relay to the destination as originally developed in [2], even though the beamforming gain resulting from this coherent transmission can significantly improve outage performance. While coherent transmission requires channel phase knowledge at the transmitter which increases the complexity of the system compared with non-coherent transmission, this phase knowledge can be obtained via the reciprocity between forward and reverse channels in time-division duplexed (TDD) systems, or via feedback from the destination in frequency-division duplexed (FDD) systems [28]. In this paper, we provide a comprehensive analysis of the outage performance of coherent $\mathrm{pDF}$ relaying and compare it with the outage performance of existing DF-based schemes in [6]-[27].

Outage performance is directly related to the achievable rate performance and can depend on whether the relay decodes the source message fully or partially. Compared with full DF relaying, partial DF (pDF) relaying requires more advanced signal processing because of message splitting and superposition coding at the source and more complex decoding at the destination. However, pDF relaying has many advantages that make it appealing for practical implementation. Partial DF (pDF) not only can achieve a better transmission rate in various half-duplex settings [29], but also can be applied in the case when some information needs privacy and is to be decoded only at the destination but not the relay. Moreover, pDF relaying can be more adaptable to the fading channel variation by only sending a sufficient part of the source information to relay such that the relay can decode this part successfully. This adaptability, however, also complicates the system as it requires enough channel state information (CSI) at the source. This paper alleviates this transmitter CSI requirement by using the CSI only to switch between direct transmission and relaying but not to adapt the rate splitting for each channel realization.

In the half-duplex mode, pDF relaying can achieve higher transmission rate than full DF relaying as shown in [29] and 
also in our previous work [30]. In many practical systems such as LTE-A [1] or GSM [31], practical constraints like fixed halfduplex phase durations can further increase the performance gap between partial and full DF relaying. In the full-duplex mode, pDF relaying achieves the same rate as the full DF relaying at the optimal resource allocation for the basic relay channel [3] but achieves a higher rate in larger networks including cooperative multiple access transmission [32], two-way relay channel (TWRC) [33] and interference channel with source cooperation [34]. Thus, outage analysis for the pDF scheme is more general and provides a foundation for the outage performance in larger networks in both half and full-duplex modes. Such analyses also cover full DF relaying as a special case, and can contrast the outage performance of coherent pDF relaying with existing full and partial DF schemes in [6]-[24], [26] to show the advantage of coherent pDF relaying.

Deriving analytical expression for the outage probability is often challenging; it is even more so for the relay channel because of multiple fading links and multiple outage events at both the relay and the destination. We can infer, nevertheless, that having analytical expression for outage results of coherent partial DF relaying is highly valuable. Such a result will allow fast, accurate outage performance evaluation without laborious and time-consuming simulations. Further, it can bring analytical insights to the factors that affect performance, such as power allocation, rate splitting and phase durations. These insights and the analytical expression results can then serve as a basis for outage analysis and optimization when integrating a relaying scheme in larger networks.

\section{B. Related Works}

Most existing works on relay outage performance are for full DF relaying with non-coherent transmission. For the basic single relay channel, outage performance has been analyzed for a variety of half-duplex full DF schemes from dual-hop to selection relaying with variable phase durations and power allocation [6]-[10]. These works consider the optimal resource allocation for minimum outage probability [6], [10] and analyze the diversity order [7]-[9]. Asymptotic outage analysis shows that half-duplex DF with fixed phase durations achieves the maximum diversity order of 2 if transmission switches between direct and relay modes depending on the outage at the relay [7]. Using variable phase durations improves the diversitymultiplexing tradeoff [9]. However, this technique requires the source to have full channel state information (CSI) of the link to the relay [7]; further, it cannot achieve the max-flow min-cut bound [8].

For the relay channel with $m$ relays, outage performance has been analyzed for multi-hop relaying, and single and multirelay selection schemes [11]-[21]. These works consider the optimal relay selection for minimum outage probability [12][16]; the outage performance over Rayleigh [14], [20] and Nakagami-m fading channels [12], [13], [21]; and effects of cochannel interference on the outage probability [17]-[19]. All of these works consider only full DF relaying but not partial DF relaying even though for fixed phase durations, partial DF relaying can outperform full DF relaying [29], [30].
The outage of partial DF relaying has received some attention in [22]-[27] where the cooperative and private information parts are encoded independently [24] or by using two-level [22] or multi-level [23] superposition coding. Both independent [24] and superposition [22] coding schemes achieve a full diversity order of 2, but the independent coding scheme achieves this full diversity only when the $\mathcal{S}-\mathcal{R}$ or $\mathcal{R}-\mathcal{D}$ link is nonfading and stronger than the other two links [24]. For the relay channel with multiple relays, using partial decoding at the relays improves the diversity-multiplexing tradeoff [26]. For pDF relaying, however, not only the coding structure between the cooperative and private parts matters, but also the coherency between $\mathcal{S}$ and $\mathcal{R}$ transmission and the decoding methods at $\mathcal{R}$ and $\mathcal{D}$ can significantly affect outage performance. For example, $\mathcal{S}$ and $\mathcal{R}$ in the superposition coding scheme of [22] perform non-coherent transmission while $\mathcal{D}$ performs sequential decoding for both information parts of $\mathcal{S}$, which reduces the achievable rate and outage performance compared to coherent $\mathcal{S}-\mathcal{R}$ transmission and joint decoding at $\mathcal{D}$. In the independent scheme of [24], $\mathcal{R}$ always decodes $\mathcal{S}$ information even when the $\mathcal{S}-\mathcal{R}$ link is very weak, which leads to a diversity order of 1 in the general case when all links experience fading. In [25], [27], we consider the multiple-access transmission where both sources employ superposition coding and cooperate using coherent $\mathrm{pDF}$ relaying to send their information to $\mathcal{D}$. We only provide outage formulation (together with transmission design and rate analysis) and numerical results without analytical outage derivation or diversity analysis. To date, there is no complete analytical derivation of the outage probability for the general coherent pDF scheme with superposition coding at $\mathcal{S}$ and joint decoding at $\mathcal{D}$.

\section{Main Results and Contributions}

In this paper, we analyze the outage probability over Rayleigh fading channels for coherent pDF relaying in the basic relay channel with a single relay for both the half- and full-duplex modes. We consider the original full-duplex pDF scheme in [2] and the time-division half-duplex pDF scheme proposed in [4] and also in our previous works [5], [30]. We extend our work in the conference version [35] by providing complete proofs for the outage theorems, extensive numerical results, full comparison with existing schemes and new analyses for the full-duplex transmission. The main differences between the considered pDF relaying schemes and other existing schemes lie in the coherent transmission between the source $(\mathcal{S})$ and the relay $(\mathcal{R})$, and the joint decoding of all information parts at the destination $(\mathcal{D})$. For this reason, we will call the considered schemes as coherent $p D F$ schemes, to distinguish with other existing schemes.

In coherent pDF relaying for either half- or full-duplex transmission, $\mathcal{S}$ splits its information into a cooperative and a private part where the cooperative part is decoded at $\mathcal{R}$ and $\mathcal{D}$ while the private part is decoded at $\mathcal{D}$ only. In the full-duplex mode, both $\mathcal{S}$ and $\mathcal{R}$ transmit all the time; whereas in the half-duplex mode, the transmission is divided into two phases, $\mathcal{S}$ transmits in both phases, and $\mathcal{R}$ listens in the first phase and transmits in the second. The cooperative part is transmitted coherently from 
both $\mathcal{S}$ and $\mathcal{R}$. Our considered half-duplex scheme is different from the pDF scheme in [22] in that $\mathcal{D}$ employs joint instead of separate decoding for the two information parts. This scheme is also different from that in [24] as it uses superposition instead of independent coding for the two information parts.

We assume full CSI at all receivers, where each receiver knows both the phases and amplitudes of the links to it. Transmitters have only limited CSI where both $\mathcal{R}$ and $\mathcal{S}$ know the phase of its respective link to $\mathcal{D}$; further, $\mathcal{S}$ knows the relative amplitude order between its links to $\mathcal{R}$ and $\mathcal{D}$ (but not necessarily the actual amplitudes) in addition to the longterm channel statistics. The phase knowledge at the transmitters allows $\mathcal{S}$ and $\mathcal{R}$ to perform coherent transmission and is a standard assumption in all coherent relaying literature [2], [4], [32]. The link order knowledge allows $\mathcal{S}$ to choose to perform either coherent transmission when the $\mathcal{S}-\mathcal{R}$ link is stronger than the $\mathcal{S}-\mathcal{D}$ link, or direct transmission in the opposite link order. The switching between coherent and direct transmissions allows the considered schemes to achieve a full diversity order of 2 even all links are fading. These transmitters CSI can be obtained either by channel reciprocity in time-division implementation, or by feedback from $\mathcal{D}$ [28]. Even though reciprocity may allow us to obtain the full channel knowledge, our analysis only requires phase knowledge at $\mathcal{S}$ and $\mathcal{R}$, and the order of link amplitudes, but not the actual amplitude values, at $\mathcal{S}$.

We provide analytical expressions for the outage probability of the coherent pDF relaying with fixed resource allocation and then obtain the optimal resource allocation numerically. The analytical expressions depend only on the channel statistics. We then use these expressions to study the impact of coherent transmission and pDF relaying on the outage probability, derive the diversity order and compare with existing schemes. These expressions also provide the basis for extending the analysis to include interference in larger network settings.

The outage analysis considers 2 transmission scenarios including pDF relaying when the relay link is stronger than the direct link and direct transmission in the opposite link order. The outage for pDF relaying takes into account the outage of the cooperative part at $\mathcal{R}$ and the outage of both cooperative and private parts at $\mathcal{D}$. Because of the superposition structure and joint decoding at $\mathcal{D}$, an outage for the cooperative part also leads to an outage for the private part. We further analyze the asymptotic outage behavior at high SNR and show that the considered coherent pDF schemes achieve a full diversity order of 2 in both half- and full-duplex modes. Comparison also shows that the coherent schemes outperform all existing schemes in outage performance. In half-duplex transmission, outages of the coherent partial and full DF schemes converge at high SNR which agrees with their rate convergence shown in [29]. However, at high target rates and low or moderate SNR, the outage performance of partial DF is better than full DF.

\section{Paper Outline}

The remainder of this paper is organized as follows. Section II presents half and full-duplex relay channel models. Section III describes the half-duplex coherent pDF scheme
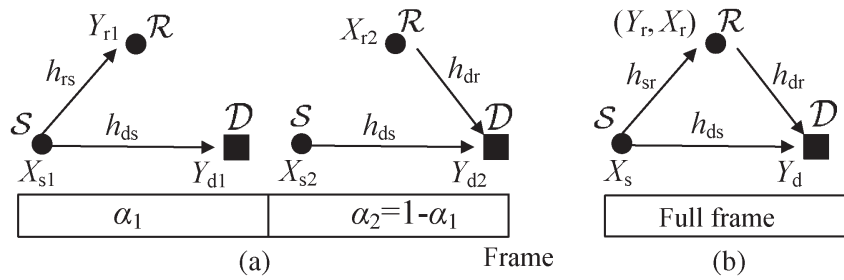

(b)

Fig. 1. Half-duplex and full-duplex models for the relay channel. (a) Halfduplex. (b) Full-duplex.

and shows its achievable rate. Section IV provides the outage probability analysis of the considered scheme and Section V shows its diversity order. Section VI compares the considered half-duplex DF scheme with existing schemes. Section VII considers the full-duplex coherent pDF scheme and its outage probability. Section VIII presents numerical results and Section IX concludes the paper.

\section{Channel Model}

Fig. 1 illustrates the full- and half-duplex relay channel models where the source $(\mathcal{S})$ wishes to communicate with the destination $(\mathcal{D})$ with help from a relay $(\mathcal{R})$ and each node has single transmit or receive antenna. While multiple-antennas or MIMO are used in many modern communication systems, single antenna implementation is still common especially in small equipments as in most of the current smartphones because of size limitation. The analyses of the single antenna systems also provide the basis for the analyses of multiple antenna systems. Next, we present the mathematical expressions for full- and half-duplex channel models.

\section{A. Half-Duplex Channel}

Fig. 1(a) illustrates the half-duplex relay channel where each node can only transmit or receive at each time. Using time division, each transmission block is divided into 2 phases and the discrete-time channel model is given as follows:

$$
\begin{aligned}
& \text { Phase 1: } Y_{r 1}=h_{r s} X_{s 1}+Z_{r 1}, Y_{d 1}=h_{d s} X_{s 1}+Z_{d 1}, \\
& \text { Phase 2: } Y_{d 2}=h_{d s} X_{s 2}+h_{d r} X_{r 2}+Z_{d 2},
\end{aligned}
$$

where $Y_{r 1}$ is the signal received by $\mathcal{R}$ during the 1st phase; $Y_{d k}, k \in\{1,2\}$ is the signal received by $\mathcal{D}$ during the $k^{\text {th }}$ phase; and all the $Z_{l}, l \in\{r 1, d 1, d 2\}$, are i.i.d complex Gaussian noises $(\mathcal{C N}(0,1)) . X_{s 1}$ and $X_{s 2}$ are the signals transmitted from $\mathcal{S}$ during the 1 st and 2 nd phases, respectively. $X_{r 2}$ is the signal transmitted from $\mathcal{R}$ in the 2 nd phase.

Each link is affected by Rayleigh fading and pathloss as follows:

$$
h_{k}=\frac{\tilde{h}_{k}}{d_{k}^{\gamma_{k} / 2}}=g_{k} e^{j \theta_{k}}, \quad k \in\{r s, d s, d r\}
$$

where $\tilde{h}_{k}$ is the small scale fading component and has a complex Gaussian distribution $(\mathcal{C N}(0,1))$. The large scale fading component is captured by a pathloss model where $d_{k}$ is the distance between two nodes and $\gamma_{k}$ is the attenuation factor. Let 
$g$ and $\theta_{k}$ be the amplitude and the phase of a link coefficient, then $g_{k}=\left|\tilde{h}_{k}\right| / d_{k}^{\gamma_{k} / 2}$ has Rayleigh distribution while $\theta_{k}$ has uniform distribution between $[0,2 \pi]$.

\section{B. Full-Duplex Channel}

When the nodes work in the full-duplex mode, the relay can transmit and receive at the same time as shown in Fig. 1(b) and the discrete-time channel model can be expressed as follows:

$$
Y_{r}=h_{r s} X_{s}+Z_{r}, Y_{d}=h_{d s} X_{s}+h_{d r} X_{r}+Z_{d}
$$

where $Y_{r}$ and $Y_{d}$ are the signals received by $\mathcal{R}$ and $\mathcal{D}$, respectively. $Z_{r}$ and $Z_{d}$, are i.i.d complex Gaussian noises $(\mathcal{C N}(0,1)) . X_{s}$ and $X_{r}$ are the signals transmitted from $\mathcal{S}$ and $\mathcal{R}$, respectively. Note that there is no phase division in the fullduplex mode, but $\mathcal{S}$ and $\mathcal{R}$ transmit during the whole frame.

In both full- and half-duplex modes, we consider block fading where the links stay constant in each transmission block through the 2 phases and change independently in the next block. We assume the following CSI: as receivers, $\mathcal{D}$ knows $h_{d s}$ and $h_{d r}$ and $\mathcal{R}$ knows $h_{r s}$; as transmitters, $\mathcal{S}$ and $\mathcal{R}$ each knows the phase of its respective link to $\mathcal{D}$, and $\mathcal{S}$ knows if the amplitude of its link to $\mathcal{D}$ is weaker or stronger than the link to $\mathcal{R}$. These CSI can be obtained via channel reciprocity or feedback as discussed in [25], [28]. The link order knowledge is important for $\mathcal{S}$ since decoding at $\mathcal{R}$ will constrain the achievable rate when the $\mathcal{S}-\mathcal{R}$ link is weaker than $\mathcal{S}-\mathcal{D}$ link. Hence, $\mathcal{S}$ only cooperates with $\mathcal{R}$ when the $\mathcal{S}-\mathcal{R}$ link is stronger than the $\mathcal{S}-\mathcal{D}$ link and performs direct transmission in the opposite link order. The phase knowledge allows coherent transmission between $\mathcal{S}$ and $\mathcal{R}$ to obtain the beamforming gain to $\mathcal{D}$ and is a standard assumption in coherent relaying literature [2], [4], [32]. As the outage probability for any scheme depends on the statistics of the channel links, $\mathcal{S}$ also needs to know the statistics of the links but not the exact instantaneous links.

We consider no interference in this model as we focus on the fundamental outage performance of pDF relaying. When deployed in a larger setting such as in an ad hoc or cellular network, the received signals at $\mathcal{R}$ and $\mathcal{D}$ will be perturbed by interference in addition to noise. While interference reduces the achievable rate and outage performance, we expect that coherent $\mathrm{pDF}$ relaying will keep outperforming the existing DF schemes in the presence of interference. The outage analyses in Sections IV and VII can be generalized to include interference in the noise terms by specifying new distributions of these terms that reflect both thermal noise and interference at $\mathcal{R}$ and $\mathcal{D}$. This topic is outside the scope of the current paper. The analysis in this paper, however, should provide the basis and set up the stage for further analysis with interference in future works.

\section{A Coherent Half-Duplex Partial DF Scheme}

The considered coherent pDF relaying scheme is a special case of the half-duplex cooperative scheme for the multiple access channel where 2 sources cooperatively send their information to a destination in our previous works [5], [25], [30]. These works analyze the rate region [5], [30] and only formulate the outage performance [25]. Here, we adapt this scheme to the relay channel with limited CSI at transmitters and analytically derive its outage performance. We briefly describe this coherent pDF scheme specifically for the relay channel to set up the stage for the outage analysis to follow. This halfduplex pDF scheme is the same as that in [4] (which is called a DF scheme in [4] but $\mathcal{R}$ decodes only part of information from $\mathcal{S})$. Moreover, this scheme can be seen as an adaptation of the full-duplex scheme in [2] to the half-duplex mode using time division [30].

Each transmission block is divided into 2 phases with normalized durations $\alpha_{1}$ and $\alpha_{2}=1-\alpha_{1}$. In each block, $\mathcal{S}$ splits its information into a cooperative part (indexed by $i$ ) and a private part (indexed by $j$ ) and encodes them using superposition coding [5]. $\mathcal{S}$ sends the private part at rate $R_{p}$ and the cooperative part at rate $R_{c}$, so that the total transmit rate is $R=R_{p}+R_{c}$. In phase $1, \mathcal{S}$ sends $i$ and $\mathcal{R}$ decodes it. Then, in phase $2, \mathcal{S}$ sends both parts $(i, j)$ and $\mathcal{R}$ forwards $i$. At the end of phase 2, $\mathcal{D}$ utilizes the received signals in both phases to decode both parts using joint maximum likelihood (ML) decoding [5].

Using Gaussian signaling, $\mathcal{S}$ and $\mathcal{R}$ construct their transmit signals in each phase as follows:

$$
\begin{aligned}
\text { Phase 1: } X_{s 1} & =\sqrt{\rho_{11}} U_{1}(i), \\
\text { Phase 2: } X_{s 2} & =\sqrt{\rho_{10}} V_{2}(j)+\sqrt{\rho_{12}} U_{2}(i), \\
X_{r 2} & =\sqrt{\rho_{22}} U_{2}(i),
\end{aligned}
$$

where $U_{1}, V_{2}$ and $U_{2}$ are i.i.d Gaussian signals $(\mathcal{N}(0,1))$, and $X_{s 2}$ is superposed on $U_{2}$. We use Gaussian signaling as it maximizes the pDF achievable rates as shown in [36]. The codeword $V_{2}$ conveys the private message. Note that $U_{1}$ and $U_{2}$ convey the same information (cooperative message) but are independent codewords (different signals from separate constellations) sent in different phases. These independent codewords simplify the analysis of the decoding at $\mathcal{D}$ by allowing the joint distribution of the ML decoding rule to factor into products as shown later in (7). Here, $\rho_{11}$ and $\rho_{10}$ are the transmission powers allocated for signals $U_{1}$ and $V_{2}$, respectively, $\rho_{12}$ and $\rho_{22}$ are the transmission powers allocated for signal $U_{2}$ by $\mathcal{S}$ and $\mathcal{R}$, respectively. These power allocation parameters satisfy the following power constraints:

$$
\alpha_{1} \rho_{11}+\alpha_{2}\left(\rho_{10}+\rho_{12}\right)=P_{s}, \rho_{22}=P_{r} / \alpha_{2} .
$$

where $P_{s}$ and $P_{r}$ are the transmit powers of $\mathcal{S}$ and $\mathcal{R}$, respectively.

Using ML decoding, $\mathcal{R}$ utilizes the received signal $Y_{r 1}$ in (1) to decode the cooperative information part $(i)$ as in the direct point-to-point transmission. It can been easily shown that $\mathcal{R}$ can reliably decodes $i$ if

$$
R_{c} \leq \alpha_{1} \log \left(1+g_{r s}^{2} \rho_{11}\right)=C_{1}
$$

At the end of phase 2, $\mathcal{D}$ also employs ML decoding and utilizes both $Y_{d 1}$ and $Y_{d 2}$ in (1) to jointly decode both information parts. 
More specifically, $\mathcal{D}$ decides that $(\hat{i}, \hat{j})$ was sent if:

$$
\begin{aligned}
& P\left(\boldsymbol{y}_{d 1}, \boldsymbol{y}_{d 2} \mid \hat{\boldsymbol{x}}_{s 1}, \hat{\boldsymbol{x}}_{s 2}, \hat{\boldsymbol{x}}_{r 2}\right) \geq P\left(\boldsymbol{y}_{d 2}, \boldsymbol{y}_{d 2} \mid \boldsymbol{x}_{s 1}, \boldsymbol{x}_{s 2}, \boldsymbol{x}_{r 2}\right) \\
& \quad \stackrel{(a)}{\Leftrightarrow} P\left(\boldsymbol{y}_{d 1} \mid \hat{\boldsymbol{x}}_{s 1, \hat{i}}\right) P\left(\boldsymbol{y}_{d 2} \mid \hat{\boldsymbol{x}}_{s 2, \hat{j}, \hat{i}}, \hat{\boldsymbol{x}}_{r 2, \hat{i}}\right) \\
& \quad \geq P\left(\boldsymbol{y}_{d 1} \mid \boldsymbol{x}_{s 1, i}\right) P\left(\boldsymbol{y}_{d 2} \mid \boldsymbol{x}_{s 2, j, i}, \boldsymbol{x}_{r 2, i}\right) \\
& \text { for all } \boldsymbol{x}_{s 1, i} \neq \hat{\boldsymbol{x}}_{s 1, \hat{i}}, \boldsymbol{x}_{s 2, j, i} \neq \hat{\boldsymbol{x}}_{s 2, \hat{j}, \hat{i}} \text { and } \boldsymbol{x}_{r 2, i} \neq \hat{\boldsymbol{x}}_{r 2, \hat{i}}
\end{aligned}
$$

where $(a)$ follows since $X_{s 1}$ is independent from $X_{s 2}$ and $X_{r 2}$. Then, by analyzing this decoding rule, a decoding error can occur for the cooperative $(i)$ or the private $(j)$ part or both. However, because of superposition coding, if $i$ is incorrectly decoded, $j$ will also be decoded incorrectly. Hence, we consider 2 cases. The first case happens when $i$ is decoded correctly, $\mathcal{D}$ then can decode $j$ from $Y_{d 2}$ after removing $U_{2}(i)$. Consequently, $Y_{d 2}$ becomes similar to the received signal in the direct transmission. Hence, the rate constraint for $j$ can be expressed as follows:

$$
R_{p} \leq \alpha_{2} \log \left(1+g_{d s}^{2} \rho_{10}\right)=C_{2}
$$

The second case happens when $\mathcal{D}$ decodes $i$ incorrectly, then $j$ will also be decoded incorrectly. Because of the joint decoding performed at $\mathcal{D}$, this incorrect decoding will result in a constraint on the total rate of both information parts. Since the cooperative part $(i)$ is sent in both phases, this rate constraint is obtained from $Y_{d 1}$ and $Y_{d 2}$ as follows:

$$
\begin{aligned}
R_{c}+R_{p} \leq & \alpha_{1} \log \left(1+\mathrm{SNR}_{1}\right)+\alpha_{2} \log \left(1+\mathrm{SNR}_{2}\right) \\
= & \alpha_{1} \log \left(1+g_{d s}^{2} \rho_{11}\right) \\
& +\alpha_{2} \log \left(1+g_{d s}^{2} \rho_{10}+\left(g_{d s} \sqrt{\rho_{12}}+g_{d r} \sqrt{\rho_{22}}\right)^{2}\right) \\
= & C_{3} .
\end{aligned}
$$

where $\mathrm{SNR}_{k}, k \in\{1,2\}$ is the $\mathrm{SNR}$ of all the received signals (due to $\mathcal{D}$ joint decoding) in phase $k$.

\section{A. Achievable Rate}

Using the constraints obtained from the ML decoding at $\mathcal{R}$ and $\mathcal{D}$, we obtain the following achievable rate:

Theorem 1: The achievable rate of the considered halfduplex coherent partial DF scheme for each channel realization is $R=R_{p}+R_{c}$, where the pair $\left(R_{p}, R_{c}\right)$ satisfies the following constraints:

$$
R \leq \min \left\{C_{1}+C_{2}, C_{3}\right\}
$$

for some $0 \leq \alpha_{1} \leq 1, \alpha_{2}=1-\alpha_{1}$ and power allocation parameters satisfying (5). The expressions for $C_{1}, C_{2}$ and $C_{3}$ are given in and (6), (8) and (9), respectively.

Proof: Obtained by combining (6), (8) and (9) with $R=$ $R_{p}+R_{c}$. For the detailed ML decoding error analysis, see Appendix A in [25].

Remark 1: We have normalized the noise powers in $C_{1}$, $C_{2}$ and $C_{3}$ as modeled in (1). Other practical factors such as transmitter and receiver antenna gains are also absorbed into the channel gain in this model. When applying in a practical system with link adaptation that uses practical modulation and coding techniques, the achievable rates can be approximated by including a SNR gap in the rate equations [37]. Such an SNR gap factor can also account for available transmit power margin. Thus the subsequent outage analysis and results are still applicable with appropriate SNR gap factors.

Remark 2: When integrating this model in a larger network, the interference from other nodes in the network will also need to be considered. For example, in a heterogeneous LTEA network, the interference on the $\mathcal{S}, \mathcal{R}$ and $\mathcal{D}$ from the other nodes and base stations in the network will be random and depend mainly on the distances between these equipments and the cooperative users. The average achievable rate of coherent pDF relaying deployed in a network is studied in [38], taking into account interference from other nodes based on stochastic geometry [39]. The outage analysis in this paper can be similarly used as a basis for future analyses of the outage performance of coherent $\mathrm{pDF}$ relaying when integrating into a larger network.

\section{B. Coherent Partial DF Relaying}

Since the power allocation parameters are design variables, we can coarsely adapt the scheme to the channel configuration to obtain two transmission cases as follows:

1) Case $1\left(g_{r s} \leq g_{d s}\right)$ : Direct transmissions.

In this case, we set $\alpha_{1}=1, \rho_{10}=\rho_{12}=\rho_{22}=0$ and let $\rho_{11}=P_{s}$. This case is identical to the classical point-to-point communication where $\mathcal{R}$ is not used and $\mathcal{S}$ sends its information directly to $\mathcal{D}$ at rate $R \leq \log \left(1+g_{d s}^{2} P_{s}\right)$.

2) Case $2\left(g_{r s}>g_{d s}\right)$ : Partial DF relaying.

In this case, $\mathcal{R}$ is used to perform $\mathrm{pDF}$ relaying with all power parts as in (5). The rate constraints for this case are given in (10).

To know which case to operate, $\mathcal{S}$ is required to know the relative order of the amplitudes of its links to $\mathcal{D}$ and $\mathcal{R}$. The combination of these two transmission cases depending on the links configuration makes up the considered half-duplex coherent pDF scheme.

\section{Half-Duplex Partial DF Outage Analysis}

The outage probability is an important criterion in wireless communication when a minimum target rate $(R)$ is to be sustained in practical wireless services. For a particular fading realization, outage occurs if the rate supported by the channel is below the target rate.

Define $\mathbb{P}_{1}$ and $\mathbb{P}_{2}$ as outage probabilities in Case 1 and 2 , respectively. For Case 1, the outage probability is obtained as in point-to-point communication but under the given condition that $g_{r s} \leq g_{d s}$. For Case 2, given that $g_{r s}>g_{d s}$, then outage can occur at $\mathcal{R}$ for the cooperative information. If there is no outage at $\mathcal{R}$, then outage can occur at $\mathcal{D}$ for the cooperative or private information or both parts. The average outage probability $\left(\overline{\mathbb{P}}_{o}\right)$ is then obtained as follows:

Theorem 2: For a given target rate $R$ with a fixed rate splitting $R=R_{p}+R_{c}$, the average outage probability $\left(\overline{\mathbb{P}}_{o}\right)$ 
of the half-duplex coherent pDF scheme with specific power allocation is given as

$$
\overline{\mathbb{P}}_{o}=\mathbb{P}_{1}+\mathbb{P}_{2}=\mathbb{P}_{1}+\mathbb{P}_{r}+\mathbb{P}_{s}+\mathbb{P}_{i}
$$

$$
\text { with } \mathbb{P}_{1}=\mathrm{P}\left[R>\log \left(1+g_{d s}^{2} \rho_{1}\right), g_{r s} \leq g_{d s}\right] \text {, }
$$

$$
\begin{aligned}
& \mathbb{P}_{r}=\mathrm{P}\left[R_{c}>C_{1}, g_{r s}>g_{d s}\right], \\
& \mathbb{P}_{s}=\mathrm{P}\left[R>C_{3}, R_{c} \leq C_{1}, g_{r s}>g_{d s}\right], \\
& \mathbb{P}_{i}=\mathrm{P}\left[R_{p}>C_{2}, R \leq C_{3}, R_{c} \leq C_{1}, g_{r s}>g_{d s}\right], \text { where }
\end{aligned}
$$

- $\mathbb{P}_{1}$ is the outage probability of direct transmission when Case 1 occurs,

- $\mathbb{P}_{r}, \mathbb{P}_{s}$ and $\mathbb{P}_{i}$ are the outage probabilities in Case 2,

- $\mathbb{P}_{r}$ is the outage probability at $\mathcal{R} ;$

- $\mathbb{P}_{s}$ is the outage probability for the sum rate at $\mathcal{D}$ when there is no outage at $\mathcal{R}$. It corresponds to the probability that both cooperative and private parts are in outage at $\mathcal{D}$;

- $\mathbb{P}_{i}$ is the outage probability for the private part at $\mathcal{D}$ when there are no outage at $\mathcal{R}$ and no outage for the sum rate at $\mathcal{D}$.

The analytical expressions for $\mathbb{P}_{1}, \mathbb{P}_{r}, \mathbb{P}_{s}$ and $\mathbb{P}_{i}$ are given in Lemmas 1, 2, 3 and 4, respectively.

Proof: See Lemmas 1-4.

Remark 3: Theorem 2 specifies the outage for fixed resource allocation (rate splitting and power allocation). We then numerically select the optimal resource allocation that minimizes the outage probability. Note that here we aim to derive analytical expressions for the outage probability in (11), but do not focus on the optimization of resources to minimize the outage probability. Results of Theorem 2, however, provide the basis for such a resource optimization problem.

\section{A. Outage Probability for Transmission Case 1}

Lemma 1: The outage probability for Case 1 is given as

$$
\begin{aligned}
\mathbb{P}_{1}= & \mathrm{P}\left[R>\log \left(1+g_{d s}^{2} P_{s}\right), g_{r s} \leq g_{d s}\right] \\
= & 1-\exp \left[-\frac{2^{R}-1}{\mu_{d s} P_{s}}\right]-\frac{\mu_{r s}}{\mu_{r s}+\mu_{d s}} \times \\
& \left(1-\exp \left[-\left(\frac{\left(2^{R}-1\right)\left(\mu_{r s}+\mu_{d s}\right)}{P_{s} \mu_{d s} \mu_{r s}}\right)\right]\right),
\end{aligned}
$$

where $\mu_{k}$ is the mean of $g_{k}^{2}$ for $k \in\{d s, r s, d r\}$.

Proof: See Appendix A.

Note that the above outage probability is different from outage of a scheme that always performs direct transmission because of the correlation between the two events: $\left(g_{r s} \leq g_{d s}\right)$ and $\left(R>\log \left(1+g_{d s}^{2} P_{s}\right)\right)$.

\section{B. Outage Probability for Transmission Case 2}

In this case, the outage can occur at $\mathcal{R}$ or $\mathcal{D}$. We first analyze the outage probability at $\mathcal{R}\left(\mathbb{P}_{r}\right)$ and $\mathcal{D}\left(\mathbb{P}_{d}\right)$ separately, then combine them to obtain the overall outage probability for this case as follows:

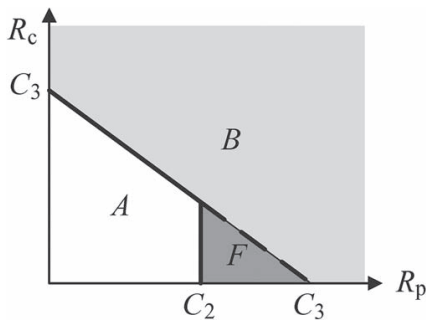

Fig. 2. Cooperative and private rate region $(A)$ and outage regions ( $B$ and $F$ ) obtained from the decoding at $\mathcal{D}$ for a given channel realization and power allocation.

Lemma 2: The outage probability for Case 2 is given as

$$
\begin{aligned}
\mathbb{P}_{2} & =\mathrm{P}\left[\text { outage at } \mathcal{R} \text { and } \mathcal{D}, g_{r s}>g_{d s}\right] \\
& =\mathbb{P}_{r}+\mathbb{P}_{d},
\end{aligned}
$$

where $\mathbb{P}_{r}=\mathrm{P}\left[\right.$ outage at $\left.\mathcal{R}, g_{r s}>g_{d s}\right]$,

$$
\mathbb{P}_{d}=\mathrm{P}\left[\text { outage at } \mathcal{D} \text {, no outage at } \mathcal{R}, g_{r s}>g_{d s}\right] .
$$

Proof: In Case 2, outage can occur at $\mathcal{R}$. If there is no outage at $\mathcal{R}$, then outage can occur at $\mathcal{D}$.

In the following sections, we set $\alpha_{1}=\alpha_{2}=1 / 2$ to simplify the analysis. Note that this assumption does not affect the diversity analysis shown later.

1) Outage at $\mathcal{R}$ : Similar to Case 1 , the outage probability at $\mathcal{R}$ can be derived as

$$
\begin{aligned}
\mathbb{P}_{r}= & \mathrm{P}\left[R_{c}>\frac{1}{2} \log \left(1+g_{r s}^{2} \rho_{11}\right), g_{r s}>g_{d s}\right] \\
= & 1-\exp \left[\frac{-\eta_{2}^{2}}{\mu_{r s}}\right] \\
& -\frac{\mu_{d s}}{\mu_{r s}+\mu_{d s}} \times\left(1-\exp \left[-\left(\frac{\eta_{2}^{2}\left(\mu_{r s}+\mu_{d s}\right)}{\mu_{d s} \mu_{r s}}\right)\right]\right),
\end{aligned}
$$

where $\eta_{2}=\sqrt{\frac{2^{2 R_{c}}-1}{\rho_{11}}}$.

2) Outage at $\mathcal{D}$ : The outage at $\mathcal{D}$ is considered when there is no outage at $\mathcal{R}$. This outage is tied directly with the decoding constraints $\left(C_{2}, C_{3}\right)$ at $\mathcal{D}$ in (10) which are repeated here for the ease of reference:

$$
R_{p} \leq C_{2}, R_{c}+R_{p} \leq C_{3} .
$$

For specific channel realization and power allocation, the rate constraints in (15) can be plotted as in Fig. 2. For a given rate splitting, there is no outage if the split rate pair is in region $A$ but there will be outage if it falls in region $B$ or $F$. We refer to the outages pertaining to regions $B$ and $F$ as the sum outage $\left(\mathbb{P}_{s}\right)$ and individual outage $\left(\mathbb{P}_{i}\right)$ respectively, since these regions are determined by the sum rate and individual rate constraints in (15). From (15), given that there is no outage at $\mathcal{R}$, the sum outage probability $\left(\mathbb{P}_{s}\right)$ is obtained as

$$
\mathbb{P}_{s}=\mathrm{P}\left[R_{c} \leq C_{1}, g_{r s}>g_{d s}\right] \mathrm{P}\left[R>C_{3} \mid R_{c} \leq C_{1}, g_{r s}>g_{d s}\right]
$$


where the first probability factor in (16) exists since we find the outage at $\mathcal{D}$ only when there is no outage at $\mathcal{R}$. Similarly, the individual outage probability $\left(\mathbb{P}_{i}\right)$ is given as

$$
\begin{aligned}
\mathbb{P}_{i}= & \mathrm{P}\left[R \leq C_{3}, R_{c} \leq C_{1}, g_{r s}>g_{d s}\right] \\
& \times \mathrm{P}\left[R_{p}>C_{2} \mid R \leq C_{3}, R_{c} \leq C_{1}, g_{r s}>g_{d s}\right],
\end{aligned}
$$

provided that there are no outage at $\mathcal{R}$ and no sum outage at $\mathcal{D}$. Then, the overall outage probability at $\mathcal{D}$ is given as

$$
\begin{aligned}
\mathbb{P}_{d}= & \mathbb{P}_{s}+\mathbb{P}_{i}=\mathrm{P}\left[R>C_{3}, R_{c}<C_{1}, g_{r s}>g_{d s}\right] \\
& +\mathrm{P}\left[R_{p}>C_{2}, R \leq C_{3}, R_{c}<C_{1}, g_{r s}>g_{d s}\right] .
\end{aligned}
$$

The analytical evaluations for these outage expressions are given in the next two Lemmas.

Lemma 3: The sum outage probability $\left(\mathbb{P}_{s}\right)$ at $\mathcal{D}$ is given as

$$
\mathbb{P}_{s}= \begin{cases}\int_{\eta_{2}}^{\eta} \int_{0}^{\beta_{3}} f_{1}\left(\beta_{1}, \zeta, \beta_{3}\right) d \beta_{1} d \beta_{3} & \\ +\exp \left[-\frac{\eta^{2}}{\mu_{r s}}\right] \int_{0}^{\eta} f_{2}\left(\beta_{1}, \zeta\right) d \beta_{1} & \text { if } \eta>\eta_{2} \\ \exp \left[-\frac{\eta_{2}^{2}}{\mu_{r s}}\right] \int_{0}^{\eta} f_{2}\left(\beta_{1}, \zeta\right) d \beta_{1}, & \text { if } \eta \leq \eta_{2}\end{cases}
$$

where $\eta_{2}$ is given in (14) and

$$
\begin{aligned}
\eta & =\sqrt{\frac{-P_{s}+\sqrt{P_{s}^{2}-\rho_{11}\left(\rho_{10}+\rho_{12}\right)\left(1-2^{2 R}\right)}}{\rho_{11}\left(\rho_{10}+\rho_{12}\right)},} \\
\zeta & =\frac{1}{\sqrt{\rho_{22}}} \sqrt{\frac{2^{2 R}}{1+\beta_{1}^{2} \rho_{11}}-\left(1+\beta_{1}^{2} \rho_{10}\right)}-\beta_{1} \sqrt{\frac{\rho_{12}}{\rho_{22}}}, \\
f_{0}(\zeta) & =1-\exp \left[-\frac{\zeta^{2}}{\mu_{d r}}\right], \\
f_{1}\left(\beta_{1}, \zeta, \beta_{3}\right) & =\frac{4 \beta_{1} \beta_{3} f_{0}(\zeta)}{\mu_{d s} \mu_{r s}} \exp \left[-\left(\frac{\beta_{1}^{2}}{\mu_{d s}}+\frac{\beta_{3}^{2}}{\mu_{r s}}\right)\right] \\
f_{2}\left(\beta_{1}, \zeta\right) & =\frac{2 \beta_{1} f_{0}(\zeta)}{\mu_{d s}} \exp \left[-\frac{\beta_{1}^{2}}{\mu_{d s}}\right] .
\end{aligned}
$$

Proof: See Appendix B

Lemma 4: The individual outage probability $\left(\mathbb{P}_{i}\right)$ at $\mathcal{D}$ is given as

$$
\mathbb{P}_{i}= \begin{cases}\mathbb{P}_{i}^{(1)} & \text { if } \eta_{2} \leq \eta \leq \eta_{1}, \quad \mathbb{P}_{i}^{(2)} \text { if } \eta_{2} \leq \eta_{1} \leq \eta, \\ \mathbb{P}_{i}^{(3)} & \text { if } \eta \leq \eta_{2} \leq \eta_{1}, \quad \mathbb{P}_{i}^{(4)} \text { if } \eta \leq \eta_{1} \leq \eta_{2}, \\ \mathbb{P}_{i}^{(5)} & \text { if } \eta_{1} \leq \eta \leq \eta_{2} \text { or } \eta_{1} \leq \eta_{2} \leq \eta\end{cases}
$$

where $\eta_{1}=\sqrt{\frac{2^{2 R_{p}}-1}{\rho_{10}}}, \eta_{2}=\sqrt{\frac{2^{2 R_{c}}-1}{\rho_{11}}}$,

$$
\begin{aligned}
\mathbb{P}_{i}^{(1)}= & \int_{\eta_{2}}^{\eta} \int_{0}^{\beta_{3}} f_{3}\left(\beta_{1}, \zeta, \beta_{3}\right) d \beta_{1} d \beta_{3} \\
& +\int_{\eta}^{\eta_{1}} \int_{\eta}^{\beta_{3}} f_{1}\left(\beta_{1}, \zeta, \beta_{3}\right) d \beta_{1} d \beta_{3} \\
& +\exp \left[-\frac{\eta^{2}}{\mu_{r s}}\right] \int_{0}^{\eta} f_{4}\left(\beta_{1}, \zeta\right) d \beta_{1}+\exp \left[-\frac{\eta_{1}^{2}}{\mu_{r s}}\right] f_{5}\left(\beta_{1}\right),
\end{aligned}
$$$$
\begin{aligned}
\mathbb{P}_{i}^{(2)}= & \int_{\eta_{2}}^{\eta_{1}} \int_{0}^{\beta_{3}} f_{3}\left(\beta_{1}, \zeta, \beta_{3}\right) d \beta_{1} d \beta_{3} \\
& +\exp \left[-\frac{\eta_{1}^{2}}{\mu_{r s}}\right] \int_{0}^{\eta_{1}} f_{4}\left(\beta_{1}, \zeta\right) d \beta_{1},
\end{aligned}
$$$$
\mathbb{P}_{i}^{(3)}=\exp \left[-\frac{\eta_{2}^{2}}{\mu_{r s}}\right]\left(\exp \left[-\frac{\eta^{2}}{\mu_{d s}}\right]+\int_{0}^{\eta} f_{4}\left(\beta_{1}, \zeta\right) d \beta_{1}\right)
$$$$
-\frac{\mu_{d s}}{\mu_{d s}+\mu_{r s}} \exp \left[-\frac{\eta_{2}^{2}\left(\mu_{r s}+\mu_{d s}\right)}{\mu_{r s} \mu_{d s}}\right]
$$$$
-\frac{\mu_{r s}}{\mu_{d s}+\mu_{r s}} \exp \left[-\frac{\eta_{1}^{2}\left(\mu_{r s}+\mu_{d s}\right)}{\mu_{r s} \mu_{d s}}\right] \text {, }
$$$$
\mathbb{P}_{i}^{(4)}=\exp \left[-\eta_{2}^{2} \mu_{r s}\right]\left(\int_{0}^{\eta} f_{4}\left(\beta_{1}, \zeta\right) d \beta_{1}+f_{5}\left(\beta_{1}\right)\right),
$$$$
\mathbb{P}_{i}^{(5)}=\exp \left[-\frac{\eta_{2}^{2}}{\mu_{r s}}\right] \int_{0}^{\eta_{1}} f_{4}\left(\beta_{1}, \zeta\right) d \beta_{1},
$$

$$
\begin{aligned}
& \text { and } f_{3}\left(\beta_{1}, \zeta, \beta_{3}\right)=\frac{4 \beta_{1} \beta_{3}}{\mu_{d s} \mu_{r s}} \exp \left[-\left(\frac{\beta_{1}^{2}}{\mu_{d s}}+\frac{\zeta^{2}}{\mu_{d r}}+\frac{\beta_{3}^{2}}{\mu_{r s}}\right)\right], \\
& f_{4}\left(\beta_{1}, \zeta\right)=\frac{2 \beta_{1}}{\mu_{d s}} \exp \left[-\left(\frac{\beta_{1}^{2}}{\mu_{d s}}+\frac{\zeta^{2}}{\mu_{d r}}\right)\right] \\
& f_{5}\left(\beta_{1}\right)=\exp \left[-\frac{\eta^{2}}{\mu_{r s}}\right]-\exp \left[-\frac{2^{2 R_{p}}-1}{\rho_{10} \mu_{r s}}\right] .
\end{aligned}
$$

Proof: See Appendix C.

\section{Half-Duplex DF AND Diversity ANALysis}

The diversity order of a transmission scheme is obtained by analyzing the asymptotic outage behavior at high SNR. Intuitively, for the outage formula in (11), the diversity order for the outage probability of the cooperative information is 2 since for the information to be lost; it always requires 2 links to be weak [7]. When the $\mathcal{S}-\mathcal{R}$ link is strong, the cooperative information will be lost if both links to $\mathcal{D}$ are weak, and when the $\mathcal{S}-\mathcal{R}$ link is weak, the cooperative information will be lost if the $\mathcal{S}-\mathcal{D}$ link is also weak. In contrary, the private information will be lost if only the direct link is weak which leads to a diversity order of 1 . Therefore, at high SNR, partial DF relaying reduces to full DF relaying to retain a diversity order of 2 . The asymptotic outage behavior at high SNR can be analyzed by applying the Taylor series expansion to the exponential function of the outage probability formula in Theorem 2. Then, 
it can be shown that all probabilities in (11) are proportional to $\mathrm{SNR}^{-2}$, except the individual outage probability for the private part which is proportional to $\mathrm{SNR}^{-1}$. Therefore, we optimally allocate zero power to the private part to deduce the asymptotic outage behavior and achieve a diversity order of 2 .

When $\mathcal{S}$ has high power, it need not split its information since it can allocate enough power in the 1 st phase to send all its information to $\mathcal{R}$. The full DF scheme therefore is the limit of the partial DF scheme as SNR increases [29], [30]. Hence, the diversity order of the partial DF scheme is the same as the DF scheme. The optimal transmission scheme reduces to the full DF at high SNR and we will derive in detail its outage probability next. The full DF scheme is obtained by removing the private information part from the partial DF scheme and has the achievable rate as follows:

Corollary 1: The achievable rate for the half-duplex coherent DF scheme is obtained from Theorem 1 by setting $R_{p}=0$, $\rho_{10}=0$ and $R=R_{c}$.

\section{A. Outage Analysis}

The full DF scheme has a simpler outage expression because of the absence of the private part outage. We use this scheme to study the asymptotic outage behavior of the pDF scheme at high SNR. The outage for the full DF scheme is obtained in a similar way to the partial DF scheme but without $\mathbb{P}_{i}$ as follows:

Corollary 2: The outage probability of the half-duplex coherent full DF scheme is given as

$$
P_{D F}=\mathbb{P}_{1}^{(D F)}+\mathbb{P}_{r}^{(D F)}+\mathbb{P}_{d}^{(D F)},
$$

where $\mathbb{P}_{1}^{(D F)}$ is the outage probability in Case 1 (direct transmission), $\mathbb{P}_{r}^{(D F)}$ is the outage probability at $\mathcal{R}$ in Case 2 (full DF relaying), and $\mathbb{P}_{d}^{(D F)}$ is the outage probability at $\mathcal{D}$ in Case 2.

Analytical expressions for $\mathbb{P}_{1}^{(D F)}, \mathbb{P}_{r}^{(D F)}$ and $\mathbb{P}_{d}^{(D F)}$ are similar to $\mathbb{P}_{1}, \mathbb{P}_{r}$ and $\mathbb{P}_{s}$ in (12), (14) and (19), respectively, with the parameter settings in Corollary 1.

\section{B. Asymptotic Outage Behavior and Diversity Order}

Without loss of generality, assume that $\mathcal{S}$ and $\mathcal{R}$ transmit power are the same $\left(P_{s}=P_{r}=P\right)$ and define a nominal SNR as the received power at the destination in direct transmission $\left(\mathrm{SNR}=\mu_{d s} P\right)$. Note that in this definition the SNR is always proportional to $P$. Next, we show that the dominant term in each outage probability is proportional to $\mathrm{SNR}^{-2}$.

Theorem 3: The asymptotic outage probability of halfduplex coherent pDF relaying at high SNR approaches the following values:

$$
\begin{aligned}
& \mathbb{P}_{1, \infty}^{(D F)}=\frac{\left(2^{R}-1\right)^{2}}{2 \mu_{d s} \mu_{r s} P^{2}}, \quad \mathbb{P}_{r, \infty}^{(D F)}=\frac{\left(2^{2 R}-1\right)^{2}}{2 \mu_{d s} \mu_{r s} P^{2}}, \\
& \mathbb{P}_{d, \infty}^{(D F)}=\frac{\delta^{2} a_{12}}{4 \mu_{d s} \mu_{d r} P^{2}},
\end{aligned}
$$

where $a_{11}=\rho_{11} / P, a_{12}=\rho_{12} / P, 0.5\left(a_{11}+a_{12}\right)=1$,

$$
\text { and } \delta=\frac{-1+\sqrt{1+a_{11} a_{12}\left(2^{2 R}-1\right)}}{a_{11} a_{12}}
$$

These expressions show that the diversity order is 2 , the maximum diversity for the basic relay channel.

Proof: The formulas in (24) are obtained using Taylor series expansion of the exponential function as shown in Appendix D.

Theorem 3 shows that the partial DF scheme achieves the maximum diversity order of 2 since it includes the full DF scheme as a special case. To achieve this diversity order, the considered scheme only requires $\mathcal{S}$ to know the relative amplitude order between its direct and cooperative links. This requirement is in contrast to the existing schemes that require $\mathcal{S}$ to have a full knowledge of the cooperative link as discussed in the following section.

\section{COMPARISON With Existing SCHEMES}

In this section, we compare the outage performance of the half-duplex coherent partial DF scheme with the existing DF [6], [7] and partial DF schemes [22], [24]. For the ease of reference, we briefly describe these schemes below, highlight their differences with our scheme and discuss their achievable rates and outage performance. We also formulate the outage probabilities for the partial DF schemes in [22] and [24] as these results have not been derived for the general channel configuration.

\section{A. DF Schemes}

1) Dual-Hop Schemes in [6]: A dual hop DF scheme is proposed in [6] as a regenerative system. In this scheme, there is no direct link from $\mathcal{S}$ to $\mathcal{D} . \mathcal{S}$ simply sends its information to $\mathcal{R}$ in phase 1 and $\mathcal{R}$ forwards it to $\mathcal{D}$ in phase 2 . The achievable rate and outage probability of this scheme are then derived in [6].

Corollary 3: The dual-hop DF scheme has a diversity order of 1 as $\mathcal{R}$ always decodes regardless of link strength and $\mathcal{D}$ receives from only one link.

2) DF Schemes in [7]: The work in [7] proposes $2 \mathrm{DF}$ schemes, derives their achievable rates and formulates their outage probabilities.

In these schemes, there is no coherent transmission between $\mathcal{S}$ and $\mathcal{R}$ since CSI is only available at receivers.

DF Scheme: The first scheme is simply called a DF scheme in which $\mathcal{S}$ transmits only in phase $1, \mathcal{R}$ always decodes regardless of the strength of the cooperative link and forwards in phase 2 , and $\mathcal{D}$ utilizes the signals in both phases to decode the transmitted information.

Corollary 4: For the non-coherent DF scheme in [7], $\mathcal{R}$ always decodes even when the cooperative link is weak. Hence, at high SNR the outage at $\mathcal{R}$ becomes dominant and leads to a diversity order of 1 [7].

Selection Relaying Scheme: The second scheme is called selection relaying scheme in which either direct transmission in two phases or non-coherent DF relaying is used. The switching between the 2 transmissions depends on the outage at $\mathcal{R}$ since $\mathcal{R}$ only forwards the information when there is no outage at $\mathcal{R}$.

Corollary 5: The selection relaying scheme achieves a full diversity order of 2 as the outage probability is not always 
constrained by the decoding at $\mathcal{R}$. However, in this scheme, $\mathcal{S}$ is required to have full CSI about the cooperative link to determine the use of direct or cooperative transmission.

In our scheme, $\mathcal{S}$ has limited CSI and it sends to $\mathcal{R}$ if the cooperative link is stronger than the direct link, but $\mathcal{S}$ does not need to know the full CSI of its link to $\mathcal{R}$. On the other hand, in our scheme, $\mathcal{S}$ and $\mathcal{R}$ have limited CSI about their direct links to $\mathcal{D}$ while they have no CSI on these links in the selection relaying scheme in [7]. Hence, depending on where CSI is available, a practical designer can choose between these two schemes. Numerical performance comparison is available in Section VIII.

\section{B. Partial DF Schemes}

This section considers the pDF schemes in [22] and [24]. The outage for the pDF scheme in [22] is formulated to calculate the expected distortion of the source while the outage formulation for the scheme in [24] assumes one of the direct links is nonfading and does not consider the general fading channel. Hence, in this section, we also formulate the outage probabilities for these $\mathrm{pDF}$ schemes.

1) Partial DF Scheme in [24]: The diversity orders for three pDF schemes are compared in [24]. Here, we consider only the first scheme as it is the most general one of these three. In this scheme, $\mathcal{S}$ splits its information into two parts as in our scheme. However, these parts are encoded independently instead of superposition coding. In phase $1, \mathcal{S}$ transmits the cooperative part to $\mathcal{R}$ and $\mathcal{D}$ while in phase $2, \mathcal{S}$ transmits the private part only and $\mathcal{R}$ forwards the cooperative part. Hence, the achievable rate for this scheme is given as follows [24]:

$$
\begin{gathered}
R_{c} \leq 0.5 \log \left(1+g_{r s}^{2} \rho_{11}\right)=A_{1}, \\
R_{p} \leq 0.5 \log \left(1+g_{d s}^{2} \rho_{10}\right)=A_{2}, \\
R_{c} \leq 0.5 \log \left(1+g_{d s}^{2} \rho_{11}+g_{d r}^{2} \rho_{2}\right)=A_{3}, \\
R_{p}+R_{c} \leq 0.5 \log \left(1+g_{d s}^{2}\left(\rho_{10}+\rho_{11}+g_{d s}^{2} \rho_{10} \rho_{11}\right)\right. \\
\left.+g_{d r}^{2} \rho_{2}\right)=A_{4},
\end{gathered}
$$

where $A_{1}$ results from the decoding at $\mathcal{R}$ and the rest $\left(A_{2}, A_{3}, A_{4}\right)$ result from the decoding at $\mathcal{D}$. $\mathcal{R}$ allocates $\rho_{2}=$ $2 P_{r}$ to the cooperative part and $\mathcal{S}$ allocates the power $\rho_{11}\left(\rho_{10}\right)$ for the cooperative (private) part such that

$$
0.5 \rho_{11}+0.5 \rho_{10}=P_{s}
$$

In [24], $\rho_{10}=\rho_{11}$ but here we make them separate variables for fair comparison with our scheme. Moreover, it is shown in [24] that this scheme achieves a diversity order of 2 when assuming that either $g_{r s}$ or $g_{d r}$ is non-fading and is stronger than the other two links.

Next, we formulate the outage probability for the pDF scheme in [24] for the general fading channel where all links are fading. This result has not been established in [24].
Corollary 6: The outage probability $\left(\mathbb{P}_{o}^{(4)}\right)$ for the noncoherent pDF scheme in [24] is given as

$$
\begin{aligned}
\mathbb{P}_{o}^{(1)} & =\mathbb{P}_{r}^{(1)}+\left(1-\mathbb{P}_{r}^{(1)}\right) \mathbb{P}_{d}^{(1)} \\
\text { where } \mathbb{P}_{r}^{(1)} & =\mathrm{P}\left[R_{c}>A_{1}\right] \\
\mathbb{P}_{d}^{(1)} & =1-\mathrm{P}\left[R_{p} \leq A_{2}, R_{c} \leq A_{3}, R_{p}+R_{c} \leq A_{4}\right]
\end{aligned}
$$

Although this pDF scheme achieves a diversity order of 2 under specific settings of some non-fading links as in [24], it has a diversity order of 1 in general fading channel where all links are fading because of the decoding requirement at $\mathcal{R}$.

Proof: $\mathbb{P}_{o}^{(1)}$ is obtained using similar formulation for $\overline{\mathbb{P}}_{o}$ in (11). The diversity order is 1 because $\mathcal{R}$ is always required to decode. At high SNR, the outage at $\mathcal{R}$ becomes dominant and $\mathbb{P}_{r}^{(1)} \approx\left(2^{2 R_{c}}-1\right)^{2} /\left(\mu_{r s} P_{s}\right)$. In [24], it is shown that the diversity order is 2 for two special cases. First, when the $\mathcal{S}-$ $\mathcal{R}$ (cooperative) link is non-fading and much stronger than the other two links such that there is no outage at $\mathcal{R}$ and the channel becomes similar to a MISO channel. Second, when the $\mathcal{R}-\mathcal{D}$ link is non-fading and much stronger than the other two links such that $A_{3}$ and $A_{4}$ are redundant $\left(R \leq \min \left\{A_{1}, A_{2}\right\}\right)$. At high SNR, $\mathbb{P}_{o}^{(1)}$ for this case is proportional to $1 / P_{s}^{2}$ [24].

2) Partial DF Scheme in [22]: The asymptotic outage behavior of a pDF scheme is studied in [22]. This pDF scheme is based on the selection relaying DF scheme in [7]. In this scheme, $\mathcal{S}$ splits its information into a cooperative and a private part, encodes them using superposition coding and sends both in phase 1 . Three transmission cases can then occur depending on the outage at $\mathcal{R}$ which tries to decode both information parts or only the cooperative part or none at all. If $\mathcal{R}$ can decode the cooperative part or both parts, it forwards what it decodes in phase 2 and $\mathcal{S}$ stays silent in this phase. If $\mathcal{R}$ cannot decode any part, $\mathcal{S}$ repeats its information in phase $2 . \mathcal{D}$ uses sequential decoding and always decodes the cooperative part first, then decodes the private part.

The rate constraints resulting from the decoding at $\mathcal{R}$ are

$$
\begin{aligned}
& R_{c} \leq 0.5 \log \left(1+\frac{g_{r s}^{2} \rho_{11}}{1+g_{r s}^{2} \rho_{10}}\right)=A_{5}, \\
& R_{p} \leq 0.5 \log \left(1+g_{r s}^{2} \rho_{10}\right)=A_{6},
\end{aligned}
$$

where $\rho_{11}$ and $\rho_{10}$ are defined as in (26). Then, the outage at $\mathcal{R}$ is given as

$$
\begin{aligned}
& \mathbb{P}_{r 1}^{(2)}=\mathrm{P}\left[R_{c}>A_{5}\right], P_{r 2}^{(2)}=\mathrm{P}\left[R_{p}>A_{6}, R_{c} \leq A_{5}\right], \\
& \mathbb{P}_{r 3}^{(2)}=\mathrm{P}\left[R_{p} \leq A_{6}, R_{c} \leq A_{5}\right],
\end{aligned}
$$

where $\mathbb{P}_{r 1}^{(2)}$ is the probability that both information parts are in outage, $\mathbb{P}_{r 2}^{(2)}$ is the probability that only the private part is in outage and $\mathbb{P}_{r 2}^{(2)}$ is the probability that there is no outage.

Hence, if there is an outage for both information parts at $\mathcal{R}$, the achievable rate at $\mathcal{D}$ satisfies

$$
\begin{aligned}
& R_{c} \leq 0.5 \log \left(1+\frac{g_{d s}^{2} \rho_{11}}{1+g_{d s}^{2} \rho_{10}}\right)=A_{7}, \\
& R_{p} \leq 0.5 \log \left(1+g_{d s}^{2} \rho_{10}\right)=A_{8} .
\end{aligned}
$$


If there is an outage for the private part only at $\mathcal{R}$, then

$$
\begin{aligned}
& R_{c} \leq 0.5 \log \left(1+\frac{g_{d s}^{2} \rho_{11}}{1+g_{d s}^{2} \rho_{10}}+g_{d r}^{2} \rho_{2}\right)=A_{9}, \\
& R_{p} \leq A_{8},
\end{aligned}
$$

where $\rho_{2}=2 P_{r}$ as defined in (25). If there is no outage at $\mathcal{R}$, then

$$
\begin{aligned}
& R_{c} \leq 0.5 \log \left(1+\frac{g_{d s}^{2} \rho_{11}+g_{d r} \rho_{22}}{1+g_{d s}^{2} \rho_{10}+g_{d r} \rho_{20}}\right)=A_{10} \\
& R_{p} \leq 0.5 \log \left(1+g_{d s}^{2} \rho_{10}+g_{d r}^{2} \rho_{20}\right)=A_{11}
\end{aligned}
$$

where $\mathcal{R}$ allocates the power $\rho_{22}$ for the cooperative part and $\rho_{20}$ for the private part. $\rho_{22}$ and $\rho_{20}$ satisfy a power constraint similar to (26).

In [22], the outage probability is obtained separately for the cooperative and private information parts and it is shown that both information parts achieve a diversity order of 2 . To find the average total outage probability; however, we jointly consider the outages for the private and cooperative parts since an outage on the cooperative part leads to an outage on the private part because of superposition coding. Similar to the selection relaying DF scheme in [7], the outage probability is formulated as follows:

Corollary 7: The outage probability for the partial DF scheme in [22] is given as

$$
\begin{aligned}
\mathbb{P}_{o}^{(2)}= & \mathbb{P}_{r 1}^{(2)}\left(\mathrm{P}\left[R_{c}>A_{7}\right]+\mathrm{P}\left[R_{p}>A_{8}, R_{c} \leq A_{7}\right]\right) \\
& +\mathbb{P}_{r 2}^{(2)}\left(\mathrm{P}\left[R_{c}>A_{9}\right]+\mathrm{P}\left[R_{p}>A_{8}, R_{c} \leq A_{9}\right]\right) \\
& +\mathbb{P}_{r 3}^{(2)}\left(\mathrm{P}\left[R_{c}>A_{10}\right]+\mathrm{P}\left[R_{p}>A_{11}, R_{c} \leq A_{10}\right]\right)
\end{aligned}
$$

where $\mathbb{P}_{r 1}^{(2)}, \mathbb{P}_{r 2}^{(2)}$ and $\mathbb{P}_{r 3}^{(2)}$ are given in (29). This scheme achieves a diversity order of 2 since it is similar to the selection relaying scheme [7]. The scheme requires $\mathcal{S}$ to have full CSI about its link to $\mathcal{R}$ to select the best transmission case.

\section{FUll-DupleX PARTIAl DF SChEME}

The partial DF scheme for the full-duplex relay channel is originally proposed in [2]. Both partial and full DF schemes achieve the same rate in Gaussian channels [3]. However, we analyze here the partial DF outage probability as it includes full DF as a special case and forms a basis for the outage analyses of other cooperative channels (cooperative MAC [32] and TWRC [33]), in which partial DF relaying can outperform full DF relaying. Furthermore, full-duplex transmission can be used in future cellular networks as many recent works have considered full-duplex transmission in wireless communication [40]-[43]. The partial DF scheme is based on superposition block Markov encoding, partial relay decoding and backward destination decoding. Note that although sliding window destination decoding leads to the same achievable rate as backward decoding in AWGN channels with much shorter decoding delay, the two decoding schemes are not equivalent in fading channels. In backward decoding [36], $\mathcal{D}$ uses its received signal in one block for each decoding step where the links are constant as we consider block fading. In sliding window decoding [2], [3], however, $\mathcal{D}$ uses its received signals in two consecutive blocks to decode where the links are different in each block which can lead to different outages. Here we consider backward decoding for the full-duplex mode.

$\mathcal{S}$ splits its information in each block into a cooperative and a private part. In block $k, \mathcal{S}$ sends the cooperative information $i^{\prime}$ of the previous block $(k-1)$ in addition to the new cooperative and private parts $(i, j)$. $\mathcal{S}$ encodes $i^{\prime}, i$ and $j$ using superposition coding. $\mathcal{R}$ decodes $i$ and forwards $i^{\prime}$ in each block. $\mathcal{D}$ uses its received signal in block $k$ to decode $i^{\prime}$ and $j$ given that it knows $i$ from the decoding in block $k+1$. Using Gaussian signaling, $\mathcal{S}$ and $\mathcal{R}$ construct their transmit signals as follows:

$$
\begin{aligned}
& X_{s}=\sqrt{\rho_{10}} V_{2}(j)+\sqrt{\rho_{11}} U_{1}(i)+\sqrt{\rho_{12}} U_{2}\left(i^{\prime}\right), \\
& X_{r}=\sqrt{P_{r}} U_{2}\left(i^{\prime}\right)
\end{aligned}
$$

where $U_{1}, V_{2}$ and $U_{2}$ are i.i.d standard Gaussian signals $(\mathcal{N}(0,1))$. The power allocation parameters are defined as in (4) and satisfy the power constraints $\rho_{11}+\rho_{10}+\rho_{12}=P_{s}$.

\section{A. Achievable Rate}

The achievable rate region is obtained by applying the results in [36] with the signals in (34) as follows:

Corollary 8: The achievable rate of the full-duplex coherent partial DF scheme in [36] for each channel realization is $R=$ $R_{p}+R_{c}$ where the rate pair $\left(R_{p}, R_{c}\right)$ satisfies the following constraints:

$$
\begin{aligned}
& R_{c} \leq \log \left(1+\frac{g_{r s}^{2} \rho_{11}}{1+g_{r s}^{2} \rho_{10}}\right)=C_{4}, \\
& R_{p} \leq \log \left(1+g_{d s}^{2} \rho_{10}\right)=C_{5}, \\
& R \leq \log \left(1+g_{d s}^{2}\left(\rho_{10}+\rho_{11}\right)\right. \\
& \left.\quad+\left(g_{d s} \sqrt{\rho_{12}}+g_{d r} \sqrt{P_{r}}\right)^{2}\right)=C_{6} .
\end{aligned}
$$

for power allocation parameters satisfying $\rho_{11}+\rho_{10}+$ $\rho_{12}=P_{s}$.

In (35), $C_{4}$ ensures the reliable decoding of $i$ at $\mathcal{R} ; C_{5}$ ensures the reliable decoding of $j$ at $\mathcal{D}$ given $i^{\prime}$ has been decoded correctly; and $C_{6}$ ensures the reliable decoding of both $i^{\prime}$ and $j$ at $\mathcal{D}$.

Similar to the half-duplex mode, we obtain 2 cases depending on the channel configuration. When $g_{r s} \leq g_{d s}$, direct transmission is chosen (Case 1); when $g_{r s}>g_{d s}$, partial DF relaying is chosen (Case 2).

\section{B. Outage Analysis}

The outage probability in a specific decoding block can be derived following similar analysis to the half-duplex scheme, assuming no outage in the previous decoding blocks in the backward decoding process. Hence, the outage probability of 
a specific block for the full-duplex scheme can be derived as in the following theorem:

Theorem 4: The outage probability for the full-duplex coherent partial DF scheme is given as follows:

$$
\mathbb{P}_{o}^{\mathrm{FD}}=\mathbb{P}_{1}^{\mathrm{FD}}+\mathbb{P}_{2}^{\mathrm{FD}}
$$

where $\mathbb{P}_{1}^{\mathrm{FD}}$ is the same as $\mathbb{P}_{1}$ in (12) while $\mathbb{P}_{2}^{\mathrm{FD}}$ is given as

$$
\mathbb{P}_{2}^{\mathrm{FD}}=\left\{\begin{array}{cl}
\mathbb{P}^{\mathrm{HD}} & \text { if } \rho_{12}>\rho_{10}\left(2^{R_{c}}-1\right) \\
1-\frac{\mu_{d s}}{\mu_{d s}+\mu_{r s}} & \text { if } \rho_{12} \leq \rho_{10}\left(2^{R_{c}}-1\right)
\end{array}\right.
$$

where $\mathbb{P}^{\mathrm{HD}}$ is similar to $\mathbb{P}_{2}$ in (13) but with the following new variable definitions:

$$
\begin{aligned}
\eta_{1} & =\sqrt{\frac{2^{R_{p}}-1}{\rho_{10}}}, \eta=\sqrt{\frac{2^{R}-1}{\rho_{12}+\rho_{10}}}, \\
\eta_{2} & =\sqrt{\frac{2^{R_{c}}-1}{\rho_{12}-\rho_{10}\left(2^{R_{c}}-1\right)}}, \\
\zeta & =\frac{1}{\sqrt{P_{r}}} \sqrt{2^{R}-\left(1+\beta_{1}\left(\rho_{12}+\rho_{10}\right)\right)}-\beta_{1} \frac{\rho_{12}}{P_{r}} .
\end{aligned}
$$

Proof: Follow a similar analysis as in the half-duplex scheme as shown in Appendix E.

The similarity between outage probabilities of the half and full-duplex schemes in (37) can be explained as follows. The achievable regions in (10) and (35) are quite similar except that in the full-duplex scheme, the constraint on the cooperative rate $R_{c}$ is affected by interference from the private signal part. This interference is not present in the half-duplex scheme, where $\mathcal{S}$ sends only the cooperative part in the 1st phase, but is present in the full-duplex scheme, where $\mathcal{S}$ sends both parts in each block. Therefore, in the full-duplex scheme, as more power is allocated to the private part, the interference at $\mathcal{R}$ increases which increases the outage probability at $\mathcal{R}$. This phenomenon occurs at low SNR or when the $\mathcal{S}-\mathcal{R}$ link is not much stronger than the $\mathcal{S}-\mathcal{D}$ link. If the condition $\left(\rho_{12} \leq \rho_{10}\left(2^{R_{c}}-1\right)\right)$ is satisfied, there will be an outage at $\mathcal{R}$ with the probability given in (37).

Corollary 9: The full-duplex partial DF scheme achieves the full diversity order of 2 .

Proof: Similar to Theorem 3, by analyzing the asymptotic outage behavior for the DF scheme.

\section{NumERICAL RESUltS}

We now provide numerical results for the outage probabilities of the considered coherent partial DF scheme to verify the derived analytical solutions. All these numerical results are for the half-duplex schemes (numerical results for the full-duplex scheme can be obtained from Theorem 4). In these simulations, all the links are Rayleigh fading channels with parameters specified in each simulation. Unless otherwise mentioned, the simulation settings in all figures including the transmit powers, pathloss attenuation factors and distances between nodes (in meters) are: $P_{s}=P_{r}=P, \gamma=2.4, d_{d s}=20, d_{d r}=15$, and $d_{r s}=7$. The average channel gain for each link is given as

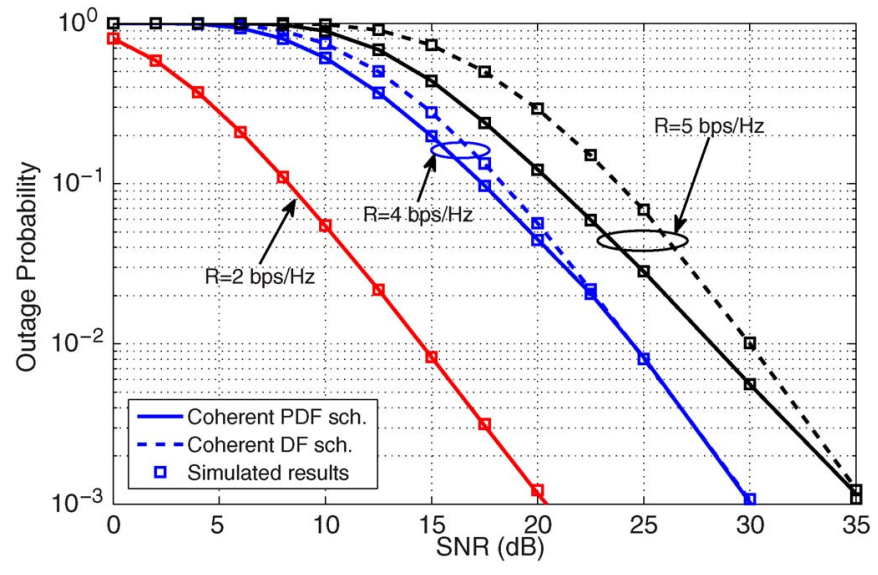

Fig. 3. Comparison between analytical results and simulations of outage probabilities for half-duplex coherent DF schemes versus SNR with different target rates $R$.

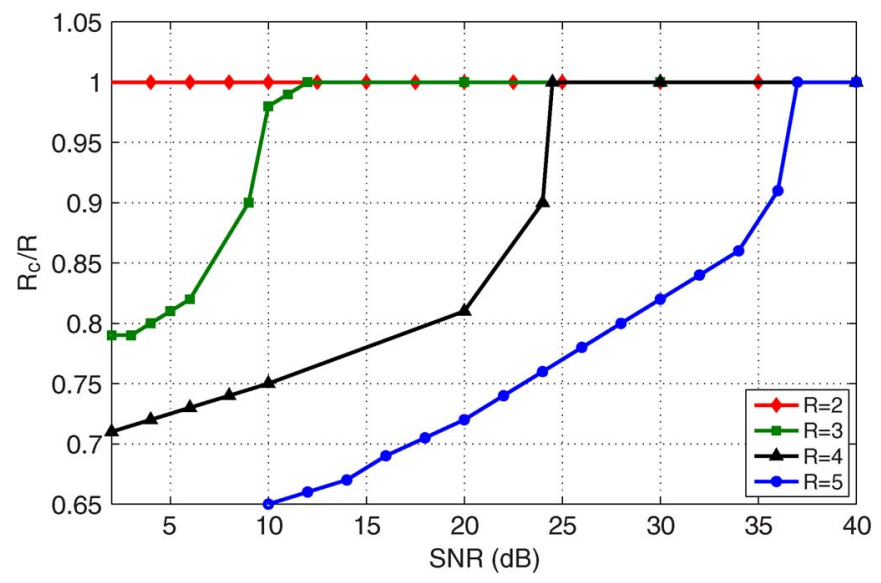

Fig. 4. Optimal ratio between cooperative rate $R_{c}$ and total rate $R$ to minimize half-duplex outage probability.

$\mu_{i j}=\frac{1}{d i j^{\gamma}}$. With these settings, we define the average received $\mathrm{SNR}$ in $\mathrm{dB}$ at $\mathcal{D}$ for the signal from $\mathcal{S}$ as follows:

$$
\mathrm{SNR}=10 \log \left(\frac{P}{d_{d s}^{\gamma}}\right) .
$$

In these simulations the optimal resource allocation parameters (power allocation and rate splitting) are varied numerically to obtain the best outage performance.

\section{A. Outage Behavior and Optimal Information Splitting}

Fig. 3 shows the outage probability versus SNR for the considered coherent DF schemes. In this figure, the simulations are obtained using $5 \times 10^{5}$ samples for each fading channel. This number of samples is sufficient for outage probabilities above $10^{-3}$ and produces the same outage results over different runs of the simulation. Results show a perfect match between analytical and simulated results. At low target rates and high SNR, partial DF and full DF perform similarly. This is because at low target rates and high SNR, $\mathcal{S}$ can send all its information to $\mathcal{R}$ without outage. However, at high target rates or low SNR, partial DF relaying outperforms full DF relaying. 


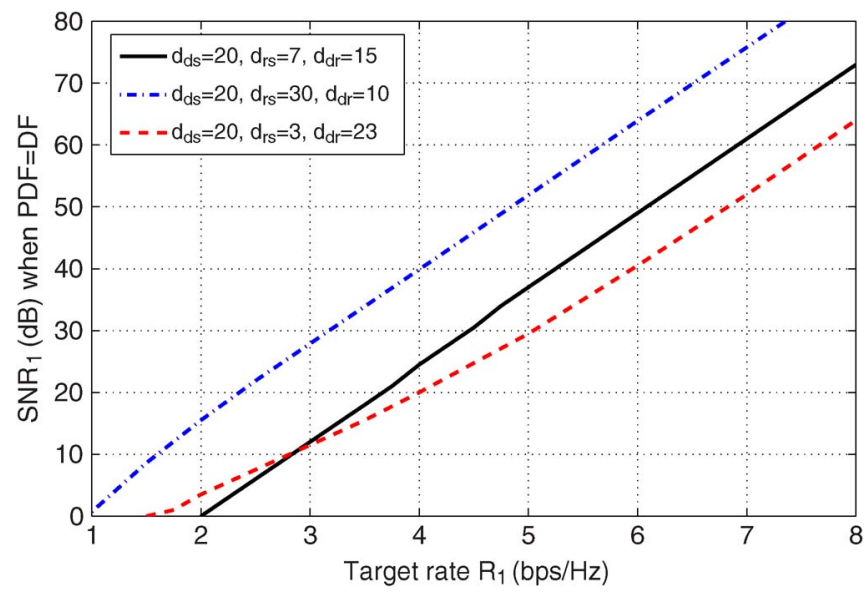

Fig. 5. Threshold $\mathrm{SNR}_{t}$ versus $R$ at which the outage probabilities for the coherent partial DF and full DF schemes coincide.

Fig. 4 shows the optimal ratio between the cooperative and the total target rates $R_{c} / R$ versus SNR. Results show that as SNR increases, $R_{c} / R$ increases till it reaches 1 . Hence, as the SNR increases, the power allocation parameters optimal for outage performance change such that the partial DF scheme becomes a full DF scheme. Moreover, as the target rate increases, the SNR threshold $\left(\mathrm{SNR}_{t}\right)$ at which the partial DF scheme becomes a full DF scheme increases.

Fig. 5 shows $\mathrm{SNR}_{t}$ versus $R$ at which partial DF becomes full $\mathrm{DF}$ for different node distance configurations as specified in the legend. It is observed that $\mathrm{SNR}_{t}$ is almost a linear function of $R$ especially at high target rates, with a slope of 12 which is insensitive to node distances. This simple relation can be appealing to the practical designers since by knowing only the SNR, they can decide between partial or full DF for the best performance. Observe also in Fig. 5 that the configuration with the longest $\mathcal{S}-\mathcal{R}\left(d_{12}\right)$ distance and hence the weakest cooperative link on the average requires the highest $\mathrm{SNR}_{t}$. In this configuration, the distance from $\mathcal{S}$ to $\mathcal{R}$ is longer than that to $\mathcal{D}$, but because of channel fading, $\mathcal{R}$ is still used from time to time. For the other two configurations, there is a cross over in $\mathrm{SNR}_{t}$ which happens because at low target rates, the relay $\mathcal{R}-\mathcal{D}$ link $\left(d_{2}\right)$ has stronger effect on outage performance than the cooperative $\mathcal{S}-\mathcal{R}$ link $\left(d_{12}\right)$, whereas the opposite holds at high target rates.

\section{B. Comparison With Existing Schemes}

Fig. 6 compares between the considered coherent partial DF scheme, the existing schemes in [6] (by analysis) [6], [7], [22], [24] (by simulation with $2 \times 10^{6}$ channel samples) and the direct transmission. Compared with the direct transmission, results show the stark gain in outage performance and diversity of the considered coherent partial and full DF schemes. Note that at low SNR, direct transmission can outperform full DF relaying but is still worse than partial DF relaying. In such a range, $\mathcal{R}$ cannot decode the whole source information since $\mathcal{S}$ has too little power for the signal to be decoded correctly at $\mathcal{R}$. On the contrary, in the partial DF scheme, $\mathcal{S}$ can split its

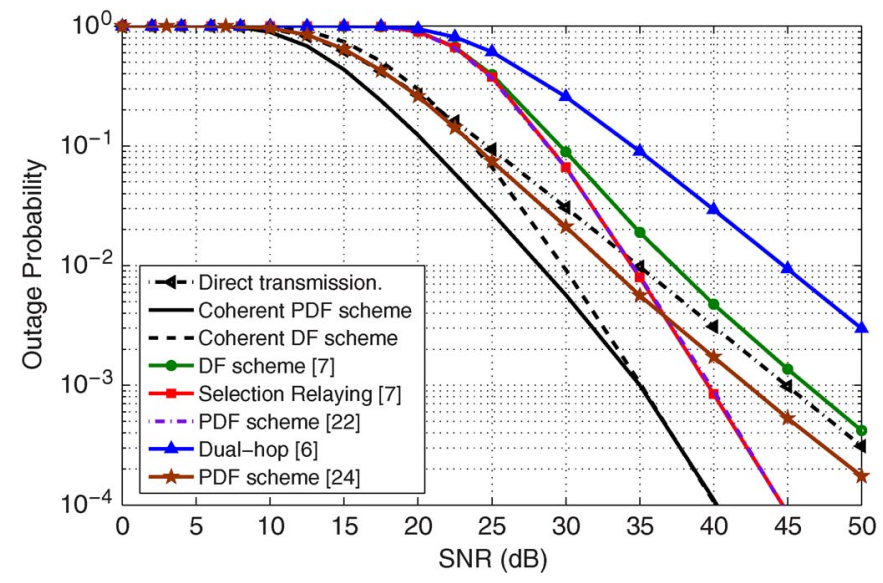

Fig. 6. Comparison between coherent DF, direct transmission and existing schemes in [6], [7], [22], [24] with $R=5 \mathrm{bps} / \mathrm{Hz}$.

information such that just enough information is sent to $\mathcal{R}$ to be decoded even at low power.

The results for the existing schemes in [6], [7], [22], [24] confirm that schemes which always require decoding at $\mathcal{R}$ achieve a diversity order of 1 since the outage at $\mathcal{R}$ becomes dominant at high SNR. The partial DF scheme in [24] outperforms the selection relaying scheme in [7] at low SNR because of rate splitting but is worse at high SNR since the outage at $\mathcal{R}$ starts dominating for the partial DF scheme. Although rate splitting and superposition coding are used in the partial DF scheme in [22], this scheme has the same outage performance of the selection relaying scheme [7] because it employs sequential decoding instead of joint decoding for the private and cooperative parts.

The selection relaying scheme in [7] and partial DF scheme in [22] both achieve a diversity order of 2 . However, these two schemes require about $4 \mathrm{~dB}$ or more power to achieve the same outage probability as the considered coherent relaying scheme. A reason is the absence of coherent transmission from $\mathcal{S}$ and $\mathcal{R}$ in these two schemes. Another factor contributing to the difference in performance is that the scheme in [22] performs sequential decoding of the cooperative and private information parts, whereas the considered schemes perform joint decoding of both parts at $\mathcal{D}$.

\section{CONCLUSION}

We have analytically derived the outage probabilities for coherent partial DF relaying in both half- and full-duplex Rayleigh fading channels. Assuming full CSI at the receivers and limited CSI at the transmitters, we take into account outages at both the relay and destination and consider outages of the cooperative and private information parts separately. We show that coherent partial DF relaying achieves the maximum diversity order of 2 in both full- and half-duplex transmissions. Analysis and numerical results show that the considered coherent partial DF relaying strategies outperform all existing decode-forward based schemes because of coherent source-relay transmission and joint decoding at the destination. Furthermore, partial DF relaying can significantly improve the outage performance over full DF relaying at low SNR and high target rates. 


\section{APPENDIX A}

\section{PROOF OF LEMMA 1}

Let $\beta_{1}=g_{d s}, \beta_{2}=g_{2}$ and $\beta_{3}=g_{r s}$. The Rayleigh distribution for any $\beta_{i}$, for $i \in\{1,2,3\}$ is given as follows:

$$
\mathrm{P}\left[\beta_{i}\right]=\frac{2 \beta_{i}}{\mu_{i}} \exp \left[-\frac{\beta_{i}^{2}}{\mu_{i}}\right] \text {. }
$$

Then, we can obtain the outage probability $\left(\mathbb{P}_{1}\right)$ for case 1 in (11) as follows:

$$
\begin{aligned}
\mathbb{P}_{1}= & \mathrm{P}\left[R>\log \left(1+\beta_{1}^{2} P_{s}\right), \beta_{3} \leq \beta_{1}\right] \\
= & \mathrm{P}\left[\beta_{1}<\sqrt{\left(2^{R}-1\right)\left(P_{s}^{-1}\right)}, \beta_{3} \leq \beta_{1}\right], \\
& =\int_{\beta_{1}=0}^{\sqrt{\frac{2^{R}-1}{P_{s}}}} \int_{\beta_{3}=0}^{\beta_{1}} \frac{4 \beta_{1} \beta_{3}}{\mu_{d s} \mu_{r s}} \exp \left[-\left(\frac{\beta_{1}^{2}}{\mu_{d s}}+\frac{\beta_{3}^{2}}{\mu_{r s}}\right)\right] d \beta_{3} d \beta_{1}, \\
& \sqrt{\frac{2^{R}-1}{P_{s}}} \\
= & \int_{\beta_{1}=0}^{\frac{2 \beta_{1}}{\mu_{d s}}} \exp \left[-\frac{\beta_{1}^{2}}{\mu_{d s}}\right]\left(1-\exp \left[-\frac{\beta_{1}^{2}}{\mu_{r s}}\right]\right) d \beta_{1} .
\end{aligned}
$$

After integrating (40), we obtain (12) in Lemma 1.

\section{APPENDIX B}

PROOF OF LEMMA 3

Starting with the expression in (18), we have

$$
\begin{aligned}
\mathbb{P}_{s} & =\mathrm{P}\left[R>C_{3}, R_{c} \leq C_{1}, g_{r s}>g_{d s}\right] \\
& =\mathrm{P}\left[\beta_{1} \leq \min \left\{\beta_{3}, \eta\right\}, \beta_{2} \leq \zeta, \beta_{3}>\eta_{2}\right] \\
& =\int_{\eta_{2}}^{\infty} \int_{\beta_{1}=0}^{\min \left\{\beta_{3}, \eta\right\}} \int_{\beta_{2}=0}^{\zeta} f_{6}\left(\beta_{1}, \beta_{2}, \beta_{3}\right) d \beta_{2} d \beta_{1} d \beta_{3}, \\
& =\int_{\eta_{2}}^{\infty} \int_{\beta_{1}=0}^{\min \left\{\beta_{3}, \eta\right\}} f_{1}\left(\beta_{1}, \zeta, \beta_{3}\right) d \beta_{1} d \beta_{3} \\
& =\left\{\begin{array}{l}
\int_{\eta_{2}}^{\eta} \int_{0}^{\beta_{3}} f_{1}\left(\beta_{1}, \zeta, \beta_{3}\right) d \beta_{1} d \beta_{3} \\
\int_{\eta}^{\infty} \int_{0}^{\eta} f_{1}\left(\beta_{1}, \zeta, \beta_{3}\right) d \beta_{1} d \beta_{3}, \quad \text { if } \eta>\eta_{2} \\
\int_{\eta_{2}}^{\infty} \int_{0}^{\eta} f_{1}\left(\beta_{1}, \zeta, \beta_{3}\right) d \beta_{1} d \beta_{3}, \quad \text { if } \eta \leq \eta_{2}
\end{array}\right.
\end{aligned}
$$

where $f_{6}\left(\beta_{1}, \beta_{2}, \beta_{3}\right)$

$$
=\frac{8 \beta_{1} \beta_{2} \beta_{3}}{\mu_{d s} \mu_{d r} \mu_{r s}} \exp \left[-\left(\frac{\beta_{1}^{2}}{\mu_{d s}}+\frac{\beta_{2}^{2}}{\mu_{d r}}+\frac{\beta_{3}^{2}}{\mu_{r s}}\right)\right] \text {. }
$$

After the integration, (41c) can be expressed as in (19).

The lower bound on $\beta_{3}$ is obtained from the second constraint in (41a) as follows:

$$
0.5 \log \left(1+\beta_{3}^{2} \rho_{11}\right)>R_{c}, \Leftrightarrow \beta_{3}>\eta_{2} .
$$

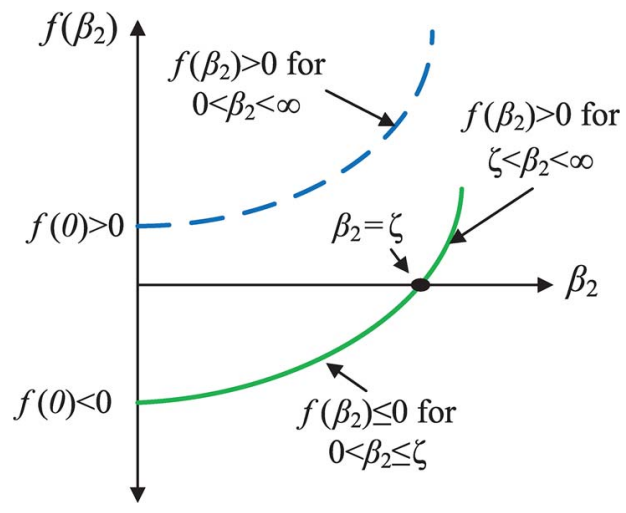

Fig. 7. The function $f\left(\beta_{2}\right)$ in (42) when $f(0)<0$ (solid-green line) and when $f(0)>0$ (dashed-blue line).

The first upper bound on $\beta_{1}$ is obtained directly from $\beta_{1}<\beta_{3}$. The second upper bound on $\beta_{1}$ and the upper bound on $\beta_{2}$ are obtained from the first constraint in (41a) as follows:

$$
\begin{aligned}
& \left(1+g_{d s}^{2} \rho_{11}\right)\left(1+g_{d s}^{2} \rho_{10}+\left(g_{d s} \sqrt{\rho_{12}}+g_{d r} \sqrt{\rho_{22}}\right)^{2}\right) \leq 2^{2 R}, \\
& \Leftrightarrow\left(\rho_{22} \beta_{2}^{2}+2 \beta_{1} \sqrt{\rho_{12} \rho_{22}} \beta_{2}+1\right. \\
& \left.\quad+\beta_{1}^{2}\left(\rho_{12}+\rho_{10}\right)-\frac{2^{2 R}}{1+\beta_{1}^{2} \rho_{11}}\right) \leq 0 \\
& \Leftrightarrow f\left(\beta_{2}\right) \leq 0 .
\end{aligned}
$$

The first derivative of $f\left(\beta_{2}\right)$ is positive $\left(\frac{\partial f\left(\beta_{2}\right)}{\partial \beta_{2}}=2 \rho_{22} \beta_{2}+\right.$ $\left.2 \beta_{1} \sqrt{\rho_{12} \rho_{22}}>0\right)$ for all values of $\left(\beta_{1}, \beta_{2}\right)$ since $\beta_{1}>0$ and $\beta_{2}>0$. Therefore, $f\left(\beta_{2}\right)$ is an increasing function of $\beta_{2}$ for $\beta_{1}>0$. Then, (42) can be satisfied only when $f(0)<0$ as shown in Fig. 7. This condition makes $f\left(\beta_{2}\right)$ negative for the interval $0 \leq \beta_{2} \leq \zeta$ (see Fig. 7), where $\zeta$ is the positive root of $f\left(\beta_{2}\right)$. However, the condition $f(0)<0$ puts another bound on $\beta_{1}$ and leads to $\beta_{1}<\eta$ since

$$
\begin{aligned}
& f(0)=1+\beta_{1}^{2}\left(\rho_{12}+\rho_{10}\right)-\frac{2^{2 R}}{1+\beta_{1}^{2} \rho_{11}} \leq 0, \\
& \Leftrightarrow \rho_{11}\left(\rho_{10}+\rho_{12}\right) \beta_{1}^{4}+2 P_{s} \beta_{1}^{2}+1-2^{2 R} \leq 0
\end{aligned}
$$

The positive root for this quadratic equation leads to $\beta_{1}<\eta$.

Now, formula (41a) is divided into two cases:

- Case 1 is obtained when $\eta>\eta_{2}$. In this case, for $\eta_{2} \leq$ $\beta_{3}<\eta$, the minimum between $\beta_{3}$ and $\eta$ is $\beta_{3}\left(\min \left\{\beta_{3}\right.\right.$, $\left.\eta\}=\beta_{3}\right)$. However, for $\beta_{3}>\eta,\left(\min \left\{\beta_{3}, \eta\right\}=\eta\right)$.

- Case 2 is obtained when $\eta \leq \eta_{2}$. In this case, $\eta$ is always the minimum between $\beta_{3}$ and $\eta$.

From these two cases, we obtain formula (19) in Lemma 3.

\section{APPENDIX C \\ Proof of LemMa 4}

Following a similar analysis to (19), we have

$$
\begin{aligned}
\mathbb{P}_{i} & =\mathrm{P}\left[R_{p}>C_{2}, R \leq C_{3}, R_{c} \leq C_{1}, \beta_{3}>\beta_{1}\right], \\
& =\mathrm{P}\left[\beta_{1} \leq \eta_{1}, R \leq C_{3}, \beta_{3}>\eta_{2}, \beta_{3}>\beta_{1}\right],
\end{aligned}
$$




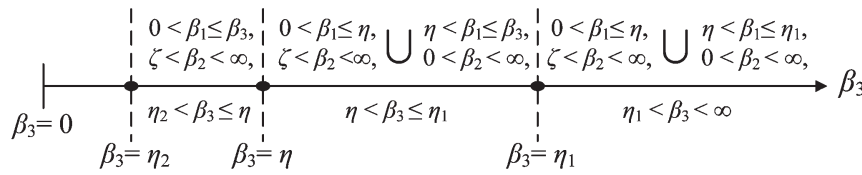

Fig. 8. The integral limits for $\mathbb{P}_{i}^{(1)}$ in (45) or for $\mathbb{P}_{i}$ in (44) when $\eta_{2}<$ $\eta \leq \eta_{1}$.

where (44) can be expressed as in (21) with

$$
\begin{aligned}
\mathbb{P}_{i}^{(1)}= & \mathrm{P}\left[\eta_{2}<\beta_{3} \leq \eta, \beta_{1} \leq \beta_{3}, \beta_{2}>\zeta\right] \\
& +\mathrm{P}\left[\eta<\beta_{3} \leq \eta_{1}, \beta_{1} \leq \eta, \beta_{2}>\zeta\right] \\
& +\mathrm{P}\left[\eta<\beta_{3} \leq \eta_{1}, \eta<\beta_{1} \leq \beta_{3}, \beta_{2}>0\right] \\
& +\mathrm{P}\left[\beta_{3}>\eta_{1}, \beta_{1} \leq \eta, \beta_{2}>\zeta\right] \\
& +\mathrm{P}\left[\beta_{3}>\eta_{1}, \eta<\beta_{1} \leq \eta_{1}, \beta_{2}>0\right], \\
\mathbb{P}_{i}^{(2)}= & \mathrm{P}\left[\eta_{2}<\beta_{3} \leq \eta_{1}, \beta_{1} \leq \beta_{3}, \beta_{2}>\zeta\right] \\
& +\mathrm{P}\left[\beta_{3}>\eta_{1}, \beta_{1} \leq \eta_{1}, \beta_{2}>\zeta\right], \\
\mathbb{P}_{i}^{(3)}= & \mathrm{P}\left[\eta_{2}<\beta_{3} \leq \eta_{1}, \beta_{1} \leq \eta, \beta_{2}>\zeta\right] \\
& +\mathrm{P}\left[\eta_{2}<\beta_{3} \leq \eta_{1}, \eta<\beta_{1} \leq \beta_{3}, \beta_{2}>0\right] \\
& +\mathrm{P}\left[\beta_{3}>\eta_{1}, \beta_{1} \leq \eta, \beta_{2}>\zeta\right] \\
& +\mathrm{P}\left[\beta_{3}>\eta_{1}, \eta \leq \beta_{1} \leq \eta_{1}, \beta_{2}>0\right], \\
\mathbb{P}_{i}^{(4)}= & \mathrm{P}\left[\beta_{3}>\eta_{2}, \beta_{1} \leq \eta, \beta_{2}>\zeta\right] \\
& +\mathrm{P}\left[\beta_{3}>\eta_{2}, \eta<\beta_{1} \leq \eta_{1}, \beta_{2}>0\right], \\
\mathbb{P}_{i}^{(5)}= & \mathbb{P}_{i}^{(6)}=\mathrm{P}\left[\beta_{3}>\eta_{2}, \beta_{1} \leq \eta_{1}, \beta_{2}>\zeta\right] .
\end{aligned}
$$

In (44), we follow the same analysis in (19) to obtain (45). However, in addition to $\beta_{1}<\beta_{3}, \beta_{1}$ in (44) has another upper bound obtained from the first constraint in (44) as follows:

$$
R_{p}>0.5 \log \left(1+\beta_{1}^{2} \rho_{10}\right), \Leftrightarrow \beta_{1}<\eta_{1}
$$

Hence, $\beta_{1} \leq \min \left\{\eta_{1}, \beta_{3}\right\}$. Moreover, the second constraint $\left(R \leq C_{3}\right)$ in (44) is the opposite to the first constraint $(R>$ $\left.C_{3}\right)$ in (41a) which means that in (42), we should have $f\left(\beta_{2}\right)>$ 0 . Hence, with help from Fig. 7, we find that $\beta_{2}$ is between

- $\zeta$ and $\infty\left(\zeta<\beta_{2} \leq \infty\right)$ when $f(0)<0$ or $\beta_{1}<\eta$.

- 0 and $\infty\left(0<\beta_{2} \leq \infty\right)$ when $f(0)>0$ or $\beta_{1}>\eta$.

This will lead to the 6 cases in (45) depending on the power allocation that determines the relative order between $\eta, \eta_{1}$ and $\eta_{2}$. Since all these cases are similarly analyzed, we only explain the limits for the first case $\left(\mathbb{P}_{i}^{(1)}\right)$ when $\eta_{2}<\eta \leq \eta_{1}$. Since $\beta_{3}>\eta_{2}$, this case can be split into 3 subcases as shown in Fig. 8:

- Subcase 1: $\eta_{2}<\beta_{3} \leq \eta$. In this case, $\beta_{3}<\eta_{1}$. Hence, $0<\beta_{1} \leq \beta_{3}$ as $\beta_{1} \leq \min \left\{\eta_{1}, \beta_{3}\right\}$. Moreover, since $\beta_{1} \leq$ $\beta_{3}$ and $\beta_{3} \leq \eta$, the range of $\beta_{2}$ is then always bigger than $\zeta\left(\beta_{2}>\zeta\right)$ to have $f\left(\beta_{2}\right)>0$ in (42). Therefore, the probability for this subcase is given as the 1 st term of $\mathbb{P}_{i}^{(1)}$.

- Subcase 2: $\eta<\beta_{3} \leq \eta_{1}$. In this case, we still have $\beta_{3}<$ $\eta_{1}$. Hence, $0<\beta_{1} \leq \beta_{3}$. However, $\beta_{3}>\eta$ which leads to two integration intervals for $\beta_{1}$ and $\beta_{2}$.
- when $0<\beta_{1} \leq \eta$, the range of $\beta_{2}$ is then always bigger than $\zeta\left(\beta_{2}>\zeta\right)$ to have $f\left(\beta_{2}\right)>0$ in (42). Therefore, the probability for this case is given as the 2nd term of $\mathbb{P}_{i}^{(1)}$.

- when $\eta<\beta_{1} \leq \beta_{3}$, the range of $\beta_{2}$ is then from 0 to $\infty$ since $f\left(\beta_{2}\right)$ is positive $\left(f\left(\beta_{2}\right)>0\right)$ for any value of $\beta_{2}>0$. Therefore, the probability for this case is given as the $3^{\text {rd }}$ term of $\mathbb{P}_{i}^{(1)}$.

- Subcase 3: $\beta_{3}>\eta_{1}$. In this case, $\beta_{3}>\eta_{1}$. Hence, $\beta_{1} \leq$ $\eta_{1}$ as $\beta_{1} \leq \min \left\{\eta_{1}, \beta_{3}\right\}$. Moreover, $\eta_{1}>\eta$ which leads to two integration intervals for $\beta_{1}$ and $\beta_{2}$.

- when $0<\beta_{1} \leq \eta$, the range of $\beta_{2}$ is then always bigger than $\zeta\left(\beta_{2}>\zeta\right)$ to have $f\left(\beta_{2}\right)>0$ in (42). Therefore, the probability for this case is given as the $4^{\text {th }}$ term of $\mathbb{P}_{i}^{(1)}$.

- when $\eta<\beta_{1} \leq \eta_{1}$, the range of $\beta_{2}$ is then from 0 to $\infty$ since $f\left(\beta_{2}\right)$ is positive $\left(f\left(\beta_{2}\right)>0\right)$ for any value of $\beta_{2}>0$. Therefore, the probability for this case is given as the 5 th term of $\mathbb{P}_{i}^{(1)}$.

By summing the probabilities of these subcases, we obtain the probability $\mathbb{P}_{i}^{(1)}$ as in (45). After simple integration, we obtain $\mathbb{P}_{i}^{(1)}$ as in (22).

\section{APPENDIX D PROOF OF THEOREM 3}

1) Asymptotic Outage Probability for Case 1: By taking the second order Taylor series expansion of the exponential functions, i.e., $\exp (-x) \approx 1-x+0.5 x^{2}$, we can then approximate $\mathbb{P}_{1}^{(D F)}$ in as follows:

$$
\begin{aligned}
\mathbb{P}_{1}^{(D F)} \approx & 2^{R}-1 \mu_{d s} P_{s}-\frac{\left(2^{R}-1\right)^{2}}{2 \mu_{d s}^{2} P_{s}^{2}} \\
& -\frac{\mu_{r s}}{\mu_{r s}+\mu_{d s}} \times\left(\frac{\left(2^{R}-1\right)\left(\mu_{r s}+\mu_{d s}\right)}{P_{s} \mu_{d s} \mu_{r s}}\right. \\
& \left.-\frac{\left(2^{R}-1\right)^{2}\left(\mu_{r s}+\mu_{d s}\right)^{2}}{2 P_{s}^{2} \mu_{d s}^{2} \mu_{r s}^{2}}\right)=\frac{\left(2^{R}-1\right)^{2}}{2 \mu_{d s} \mu_{r s} P_{s}^{2}} .
\end{aligned}
$$

This expression becomes the limit at high SNR.

2) Asymptotic Outage Probability for Case 2: We separately consider the outages at $\mathcal{R}$ and $\mathcal{D}$.

At $\mathcal{R}$ : Following similar analysis in (46), we obtain $P_{m}^{(D F)}$ as in (24).

At $\mathcal{D}$ : Before analyzing $\mathbb{P}_{d, \infty}^{(D F)}$ in (24), we assume that $P_{s}=P_{r}$ and set the following definitions:

$$
a_{11}=\rho_{11} / P_{s}, a_{12}=\rho_{12} / P_{s}, \rho_{22}=2 P_{r}
$$

where $0.5\left(a_{11}+a_{12}\right)=1$ to satisfy the power constraint in (5). 
Then, as $P_{s} \rightarrow \infty$, we approximate $\eta$ and $\zeta$ as follows:

$$
\begin{aligned}
& \eta=\sqrt{\frac{\delta}{P_{s}}}, \text { where } \delta=\frac{-1+\sqrt{1+a_{11} a_{12}\left(2^{2 R}-1\right)}}{a_{11} a_{12}}, \\
& \zeta=-\beta_{1} \sqrt{\frac{a_{12}}{2}}+\frac{1}{\beta_{1} P_{s}} \sqrt{\frac{2^{2 R}}{2 a_{11}}}
\end{aligned}
$$

Now, by taking the first order Taylor series expansion of the exponential functions, we have

$$
\begin{aligned}
& \mathbb{P}_{d, \infty}^{(D F)}
\end{aligned}
$$

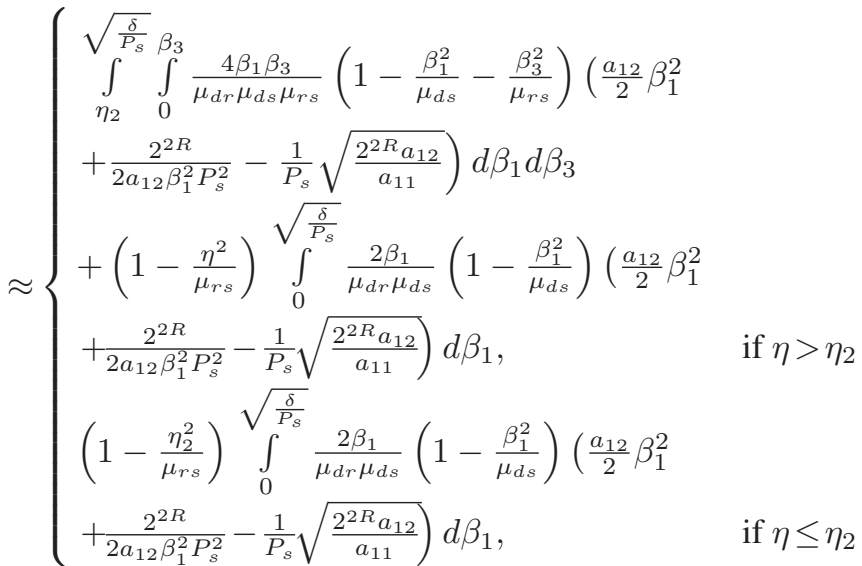$$
\approx\left\{\begin{array}{l}
\int_{\eta_{2}}^{\sqrt{\frac{\delta}{P_{s}}}} \int_{0} \frac{2 a_{12} \beta_{1}^{3} \beta_{3}}{\mu_{d r} \mu_{d s} \mu_{r s}}\left(1-\frac{\beta_{1}^{2}}{\mu_{d s}}-\frac{\beta_{3}^{2}}{\mu_{r s}}\right) d \beta_{1} d \beta_{3} \\
+\left(1-\frac{\eta^{2}}{\mu_{r s}}\right) \int_{0}^{\sqrt{\frac{\delta}{P_{s}}}} \frac{a_{12} \beta_{1}^{3}}{\mu_{d r} \mu_{d s}}\left(1-\frac{\beta_{1}^{2}}{\mu_{d s}}\right) d \beta_{1}, \quad \eta>\eta_{2} \\
\left(1-\frac{\eta_{2}^{2}}{\mu_{r s}}\right) \int_{0}^{\sqrt{\frac{\delta}{P_{s}}}} \frac{a_{12} \beta_{1}^{3}}{\mu_{d r} \mu_{d s}}\left(1-\frac{\beta_{1}^{2}}{\mu_{d s}}\right) d \beta_{1}, \quad \eta \leq \eta_{2}
\end{array}\right.
$$$$
\approx\left\{\begin{array}{l}
\sqrt{\frac{\delta}{P_{s}}} \beta_{3} \\
\int_{\eta_{2}} \int_{0} \frac{2 a_{12} \beta_{1}^{3} \beta_{3}}{\mu_{d r} \mu_{d s} \mu_{r s}} d \beta_{1} d \beta_{3} \\
+\left(1-\frac{\eta^{2}}{\mu_{r s}}\right) \int_{0}^{\sqrt{\frac{\delta}{P_{s}}}} \frac{a_{12} \beta_{1}^{3}}{\mu_{d r} \mu_{d s}} d \beta_{1}, \quad \text { if } \eta>\eta_{2} \\
\left(1-\frac{\eta_{2}^{2}}{\mu_{r s}}\right) \int_{0}^{\sqrt{\frac{\delta}{P_{s}}}} \frac{a_{12} \beta_{1}^{3}}{\mu_{d r} \mu_{d s}} d \beta_{1}, \quad \text { if } \eta \leq \eta_{2}
\end{array}\right.
$$$$
= \begin{cases}\frac{a_{12}}{12 \mu_{d s} \mu_{r s} P_{s}^{3}}\left(\delta^{3}-\frac{\left(2^{2 R}-1\right)^{3}}{a_{11}^{3}}\right) & \\ +\frac{\delta^{2} a_{12}}{4 \mu_{d s} \mu_{d r} P_{s}^{2}}, & \text { if } \eta>\eta_{2} \\ \frac{\delta^{2} a_{12}}{4 \mu_{d s} \mu_{d r} P_{s}^{2}}, & \text { if } \eta \leq \eta_{2}\end{cases}
$$

With $P_{s} \rightarrow \infty$, we obtain $\mathbb{P}_{d, \infty}^{(D F)}$ as in (24). Then, formulas (46) and (49) show that the proposed scheme achieves a diversity of order 2.

\section{APPENDIX E \\ PROOF OF THEOREM 4}

The outage in Case 1 is identical to that of the half-duplex scheme since direct transmission is optimal in this case. For Case 2, the outage analysis is also similar to the half-duplex scheme. Hence, we derive the outage at $\mathcal{R}$ only $\mathbb{P}_{o}(R)$. Let $\beta_{1}=g_{d s}$ and $\beta_{3}=g_{r s}$

$$
\begin{aligned}
\mathbb{P}_{o}(R) & =\mathrm{P}\left[R_{c}>\log \left(1+\frac{\beta_{3}^{2} \rho_{12}}{1+\beta_{3}^{2} \rho_{10}}\right), \beta_{1} \leq \beta_{3}\right] \\
& =\mathrm{P}\left[f_{6}\left(\beta_{3}\right)<0, \beta_{3} \leq \beta_{1}\right], \\
\text { where } \quad f_{6}\left(\beta_{3}\right) & =\beta_{3}^{2}\left(\rho_{12}-\rho_{10}\left(2^{R_{c}}-1\right)\right)-\left(2^{R_{c}}-1\right)
\end{aligned}
$$

Now, since $\frac{\partial f_{6}\left(\beta_{3}\right)}{\partial \beta_{3}}=2 \beta_{3}\left(\rho_{12}-\rho_{10}\left(2^{R_{c}}-1\right)\right), f_{6}\left(\beta_{3}\right)$ is an increasing function of $\beta_{3}$ if $\rho_{12}>\rho_{10}\left(2^{R_{c}}-1\right)$ and a decreasing function if $\rho_{12} \leq \rho_{10}\left(2^{R_{c}}-1\right)$. Since $f_{6}(0)=-\left(2^{R_{c}}-\right.$ 1) $\leq 0$,

- $f_{6}\left(\beta_{3}\right)<0$ if $\rho_{12}>\rho_{10}\left(2^{R_{c}}-1\right)$ and $0<\beta_{3} \leq \eta_{2}$.

- $f_{6}\left(\beta_{3}\right)<0$ if $\rho_{12} \leq \rho_{10}\left(2^{R_{c}}-1\right)$ and $0<\beta_{3} \leq \infty$.

Applying these limits into (50) and following similar analysis in Appendix A, we obtain

$$
\begin{aligned}
& \mathbb{P}_{o}(R)= \\
& \begin{cases}1-\exp \left[-\frac{\eta_{2}^{2}}{\mu_{r s}}\right]-\frac{\mu_{d s}}{\mu_{r s}+\mu_{d s}} \\
\times\left(1-\exp \left[-\left(\frac{\eta_{2}^{2}\left(\mu_{r s}+\mu_{d s}\right)}{\mu_{d s} \mu_{r s}}\right)\right]\right) & \text { if } \rho_{12}>\rho_{10}\left(2^{R_{c}}-1\right) \\
1-\frac{\mu_{d s}}{\mu_{d s}+\mu_{r s}}, & \text { if } \rho_{12} \leq \rho_{10}\left(2^{R_{c}}-1\right)\end{cases}
\end{aligned}
$$

which is the same as in the half-duplex scheme but with different $\eta_{2}$ and the additional outage when $\rho_{12} \leq \rho_{10}\left(2^{R_{c}}-1\right)$. Moreover, note that when $\rho_{12} \leq \rho_{10}\left(2^{R_{c}}-1\right)$, we obtain $R_{c}>$ $C_{4}$. Since the outage for either cooperative or private part at $\mathcal{D}$ requires $R_{c} \leq C_{4}$, the outage probability at $\mathcal{D}$ when $\rho_{12} \leq$ $\rho_{10}\left(2^{R_{c}}-1\right)$ is equal to 0 . Hence, the total outage probability is as given in Theorem 4 .

\section{REFERENCES}

[1] K. Doppler, M. Rinne, C. Wijting, C. Ribeiro, and K. Hugl, "Device-todevice communication as an underlay to LTE-advanced networks," IEEE Commun. Mag., vol. 47, no. 12, pp. 42-49, Dec. 2009.

[2] T. M. Cover and A. El Gamal, "Capacity theorems for the relay channel," IEEE Trans. Inf. Theory, vol. 25, no. 5, pp. 572-584, Sep. 1979.

[3] A. El Gamal, M. Mohseni, and S. Zahedi, "Bounds on capacity and minimum energy-per-bit for awgn relay channels," IEEE Trans. Inf. Theory, vol. 52, no. 4, pp. 1545-1561, Apr. 2006.

[4] A. Host-Madsen and J. Zhang, "Capacity bounds and power allocation for wireless relay channels," IEEE Trans. Inf. Theory, vol. 51, no. 6, pp. 2020-2040, Jun. 2005.

[5] A. Abu Al Haija and M. Vu, "A half-duplex cooperative scheme with partial decode-forward relaying," in Proc. IEEE ISIT, Aug. 2011, pp. 18861890.

[6] M. O. Hasna and M. S. Alouini, "Optimal power allocation for relayed transmissions over Rayleigh fading channels," in Proc. IEEE VTC, Apr. 2003, pp. 2461-2465.

[7] J. Laneman, D. Tse, and G. Wornell, "Cooperative diversity in wireless networks: Efficient protocols and outage behavior," IEEE Trans. Inf. Theory, vol. 50, no. 12, pp. 3062-3080, Dec. 2004.

[8] A. S. Avestimehr and D. Tse, "Outage capacity of the fading relay channel in the low-SNR regime," IEEE Trans. Inf. Theory, vol. 53, no. 4, pp. 14011415, Apr. 2007. 
[9] K. Azarian, H. El Gamal, and P. Schniter, "On the achievable diversitymultiplexing tradeoff in half-duplex channels," IEEE Trans. Inf. Theory, vol. 51, no. 12, pp. 4152-4172, Dec. 2005.

[10] D. Gunduz and E. Erkip, "Opportunistic cooperation by dynamic resource allocation," IEEE Trans. Wireless Commun., vol. 6, no. 4, pp. 1446-1454, Apr. 2007.

[11] J. Boyer, D. D. Falconer, and H. Yanikomeroglu, "Multi-hop diversity in wireless relaying channels," IEEE Trans. Commun., vol. 52, no. 10, pp. 1820-1830, Oct. 2004.

[12] D. S. Michalopoulos and G. K. Karagiannidis, "Performance analysis of single relay selection in Rayleigh fading," IEEE Trans. Wireless Commun., vol. 7, no. 10, pp. 3718-3724, Oct. 2008.

[13] M. Benjillali and M. S. Alouini, "Partner cooperation with decode-andforward: Closed-form outage analysis and comparison," IEEE Trans. Veh. Technol., vol. 62, no. 1, pp. 127-139, Jan. 2013.

[14] B. Maham, A. Behnad, and M. Debbah, "Analysis of outage probability and throughput for half-duplex hybrid-arq relay channels," IEEE Trans. Veh. Technol., vol. 61, no. 7, pp. 3061-3070, Sep. 2012.

[15] J. Luo, R. S. Blum, L. J. Cimini, L. J. Greenstein, and A. M. Haimovich, "Decode-and-forward cooperative diversity with power allocation in wireless networks," IEEE Trans. Wireless Commun., vol. 6, no. 3, pp. 793799, Mar. 2007.

[16] N. C. Beaulieu and J. Hu, "A closed-form expression for the outage probability of decode-and-forward relaying in dissimilar Rayleigh fading channels," IEEE Commun. Lett., vol. 10, no. 12, pp. 813-815, Dec. 2006.

[17] D. Lee and J. H. Lee, "Outage probability of decode-and-forward opportunistic relaying in a multicell environment," IEEE Trans. Veh. Technol., vol. 60, no. 4, pp. 1925-1930, May 2011.

[18] H. Yu, I. H. Lee, and G. L. Stuber, "Outage probability of decodeand-forward cooperative relaying systems with co-channel interference," IEEE Trans. Wireless Commun., vol. 11, no. 1, pp. 266-274, Jan. 2012.

[19] N. Suraweera and N. C. Beaulieu, "Outage probability of decode-andforward relaying with optimum combining in the presence of co-channel interference and Nakagami fading," IEEE Wireless Commun. Lett., vol. 2, no. 5, pp. 495-498, Oct. 2013.

[20] Y. Zhao, R. Adve, and T. J. Lim, "Outage probability at arbitrary SNR with cooperative diversity," IEEE Commun. Lett., vol. 9, no. 8, pp. 700702, Aug. 2005.

[21] H. A. Suraweera, P. J. Smith, and J. Armstrong, "Outage probability of cooperative relay networks in nakagami-m fading channels," IEEE Commun. Lett., vol. 10, no. 12, pp. 834-836, Dec. 2006.

[22] M. Yuksel and E. Erkip, "Broadcast strategies for the fading relay channel," in Proc. IEEE Mil. Commun. Conf., 2004, vol. 2, pp. 1060-1065.

[23] J. S. Wang, Y. H. Kim, I. Song, P. C. Cosman, and L. B. Milstein, "Cooperative relaying of superposition coding with simple feedback for layered source transmission," IEEE Trans. Commun., vol. 61, no. 11, pp. 44484461, Nov. 2013.

[24] R. U. Nabar, H. Bolcskei, and F. W. Kneubuhler, "Fading relay channels: Performance limits and space-time signal design," IEEE J. Sel. Areas Commun., vol. 22, no. 6, pp. 1099-1109, Aug. 2004.

[25] A. Abu Al Haija and M. Vu, "Spectral efficiency and outage performance for hybrid D2D-infrastructure uplink cooperation," IEEE Trans. Wireless Commun, to be published.

[26] C. Hucher, G. Othman, and A. Saadani, "A new incomplete decode-andforward protocol," in Proc. IEEE WCNC, 2008, pp. 565-569.

[27] A. Abu Al Haija and M. Vu, "Outage analysis for uplink mobile-to-mobile cooperation," in Proc. IEEE GLOBECOM, Dec. 2013, pp. 579-584.

[28] R. Mudumbai, D. Brown, U. Madhow, and H. Poor, "Distributed transmit beamforming: Challenges and recent progress," IEEE Commun. Mag., vol. 47, no. 2, pp. 102-110, Feb. 2009.

[29] L. Ong, S. Johnson, and C. Kellett, "The half-duplex AWGN singlerelay channel: Full decoding or partial decoding?" IEEE Trans. Commun., vol. 60 , no. 11, pp. 3156-3160, Nov. 2012.

[30] A. Abu Al Haija and M. Vu, "Rate maximization for half-duplex multiple access with cooperating transmitters," IEEE Trans. Commun., vol. 61, no. 9, pp. 3620-3634, Sep. 2013.

[31] T. S. Rappaport, Wireless Communications: Principles and Practice, 2nd ed. Englewood Cliffs, NJ, USA: Prentice-Hall, 2002.

[32] A. Sendonaris, E. Erkip, and B. Aazhang, "User cooperation diversity. Part I. System description," IEEE Trans. Commun., vol. 51, no. 11, pp. 1927-1938, Nov. 2003.
[33] P. Zhong and M. Vu, "Partial decode-forward coding schemes for the Gaussian two-way relay channel," in Proc. IEEE ICC, Jun. 2012, pp. 2451-2456.

[34] S. Yang and D. Tuninetti, "Interference channel with generalized feedback (a.k.a. with source cooperation): Part I: Achievable region," IEEE Trans. Inf. Theory, vol. 57, no. 5, pp. 2686-2710, May 2011.

[35] A. Abu Al Haija and M. Vu, "Outage analysis for half-duplex partial decode-forward relaying over fading channel," in Proc. IEEE Conf. GLOBECOM, Dec. 2014, pp. 1722-1727.

[36] R. El Gamal and Y.-H. Kim, Network Information Theory, 1st ed Cambridge, U.K.: Cambridge Univ. Press, 2011.

[37] J. M. Cioffi, Advanced Digital Communication-EE397C Class Notes. Stanford, CA, USA: Stanford Univ. Press, 2004

[38] H. ElKotby and M. Vu, "Interference and throughput analysis of uplink user-assisted relaying in cellular networks," in Proc. IEEE 25th Int. Symp. PIMRC, Sep. 2014.

[39] T. D. Novlan, H. S. Dhillon, and J. G. Andrews, "Analytical modeling of uplink cellular networks," IEEE Trans. Wireless Commun., vol. 12, no. 6, pp. 2669-2679, Jun. 2013.

[40] A. Sabharwal et al., "In-band full-duplex wireless: Challenges and opportunities," IEEE J. Sel. Areas Commun., vol. 32, no. 9, pp. 1637-1652, Sep. 2014.

[41] E. Ju, H. Eunsung Oh, and D. Daesik Hong, "Catching resource-devouring worms in next-generation wireless relay systems: Two-way relay and fullduplex relay," IEEE Commun. Mag., vol. 47, no. 9, pp. 58-65, Sep. 2009.

[42] M. Duarte, C. Dick, and A. Sabharwal, "Experiment-driven characterization of full-duplex wireless systems," IEEE Trans. Wireless Commun., vol. 11 , no. 12 , pp. $4296-4307$, Dec. 2012.

[43] Y.-S. Choi and H. Shirani-Mehr, "Simultaneous transmission and reception: Algorithm, design and system level performance," IEEE Trans. Wireless Commun., vol. 12, no. 12, pp. 5992-6010, Dec. 2013.

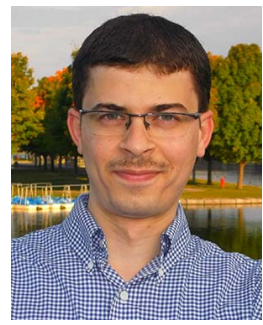

Ahmad Abu Al Haija (S'10) received the B.Sc. and M.Sc. degrees in electrical engineering from Jordan University of Science and Technology (JUST), Irbid, Jordan, in 2006 and 2009, respectively. He has been working toward the Ph.D. degree since January 2010 at McGill University, Montreal, Canada. Between February 2013 and August 2014, he was a Visiting Student at Tufts University, Medford, MA. His research interest includes cooperation in multiuser channels, resource allocation for cooperative communication systems, wireless communications, and performance analysis and evaluation over fading channels.

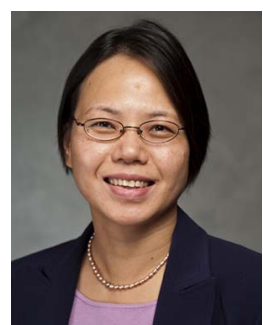

Mai Vu (M'06-SM'13) received the Ph.D. degree in electrical engineering from Stanford University, USA, after receiving the M.S.E. degree in electrical engineering from the University of Melbourne, Australia, and a bachelor's degree in computer systems engineering from the Royal Melbourne Institute of Technology (RMIT University), Australia. Between 2006 and 2008, she worked as a Lecturer and Researcher at the School of Engineering and Applied Sciences, Harvard University. During 2009-2012, she was an Assistant Professor in electrical and computer engineering at McGill University. Since January 2013, she has been an Associate Professor in the Department of Electrical and Computer Engineering at Tufts University.

Dr. Vu conducts research in wireless systems, signal processing, and networked communications. She has published extensively in the areas of cooperative and cognitive communications, relay networks, MIMO capacity and precoding, and energy-efficient communications. She has served on the technical program committee of numerous IEEE conferences and is currently an editor for the IEEE TRANSACTIONS ON WIRELESS COMMUNICATIONS. 